Report No. BMI-1503

UC-32 Mathematics and Computers (TID-4500, 16th Ed.)

Contract No. W-7405-eng -92

NUMERICAL SOLUTION OF REACTOR STRESS PROBLEMS

by

Robert F. Redmond

Lewis E. Hulbert

Richard W. Clark

February 28, 1961

BATTELLE MEMORIAL INSTITUTE

$505 \mathrm{King}$ Avenue

Columbus 1, Ohio 


\section{DISCLAIMER}

This report was prepared as an account of work sponsored by an agency of the United States Government. Neither the United States Government nor any agency Thereof, nor any of their employees, makes any warranty, express or implied, or assumes any legal liability or responsibility for the accuracy, completeness, or usefulness of any information, apparatus, product, or process disclosed, or represents that its use would not infringe privately owned rights. Reference herein to any specific commercial product, process, or service by trade name, trademark, manufacturer, or otherwise does not necessarily constitute or imply its endorsement, recommendation, or favoring by the United States Government or any agency thereof. The views and opinions of authors expressed herein do not necessarily state or reflect those of the United States Government or any agency thereof. 


\section{DISCLAIMER}

Portions of this document may be illegible in electronic image products. Images are produced from the best available original document. 


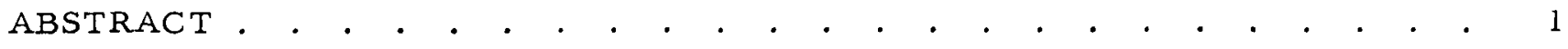

INTRODUCTION . . . . . . . . . . . . . . . . . . . . . . 1

MATHEMATICAL DESCRIPTION . . . . . . . . . . . . . . . . 4

NUMERICAL DESCRIPTION . . . . . . . . . . . . . . . . . . 8

NUMERICAL SOLUTION. . . . . . . . . . . . . . . . . . . . 12

COMPUTER CODES . . . . . . . . . . . . . . . . . . . . . 13

Matrix-Calculation Code (I) . . . . . . . . . . . . . . . . . 14

Matrix-Transpose Code (II) . . . . . . . . . . . . . . . . . . . . . . 17

Iteration Code (III) . . . . . . . . . . . . . . . . . . . . . . . . . . 17

Stress-Computation Code (IV) . . . . . . . . . . . . . . . . . . 18

Displacement-Computation Code (V) . . . . . . . . . . . . . . 18

Interpolation Code (VI). . . . . . . . . . . . . . . . . . . . . . . . . . 19

GEL 1 Input Code (VII). . . . . . . . . . . . . . . . . . . . . . . 19

COMBINING CODES . . . . . . . . . . . . . . . . . . . . . . . . 19

DISCUSSION OF RESULTS . . . . . . . . . . . . . . . . . . . 20

REFERENCES . . . . . . . . . . . . . . . . . . . . 28

APPENDIX A

MINIMIZAtion OF RESIDUAL AS AN IteRATIVE METHOD . . . . . . . A-1

APPENDIX B

DETAILS OF COMPUTER CODES

$\mathrm{B}-1$

APPENDIX C

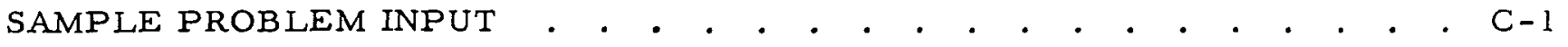




\title{
NUMERICAL SOLUTION OF REACTOR STRESS PROBLEMS
}

\author{
Robert F. Redmond, Lewis E. Hulbert, and Richard W. Clark
}

\begin{abstract}
Generalized computer codes were devised for solving stress problems of some complexity. These codes were applied to stress problems relating to the graphite moderator elements in the Experimental Gas-Cooled Reactor. The stress relief obtained by subdividing the moderator elements was evaluated. The distortion and bending moments of the elements were also determined.
\end{abstract}

\section{INTRODUCTION}

In a previous report $(1)$ the thermal-stress problem for plane strain was considered. A numerical method of solution for the plane-strain problem was described and applied to several reactor fuel-element designs. This report covers work performed for Oak Ridge National Laboratory in connection with the Experimental Gas-Cooled Reactor (EGCR). (2)

In this second report some improvements and generalizations of the previously developed computer codes are described. In addition these improved and augmented codes have been applied to the solution of a stress problem related to the fuel-element design for the EGCR. These applications and the resulting numerical solutions are also discussed.

The fuel-element assembly for the EGCR is of circular cross section. The fuel material is $\mathrm{UO}_{2}$ in the form of cored cylindrical pellets which are contained in a stainless steel tube. A cylindrical graphite sleeve encloses an assembly of seven fuel tubes with space for helium cooling gas to pass. The graphite sleeves then fit into graphite moderator pieces, one of which is shown in cross section in Figure 1. Stresses are induced by fast-neutron damage to these graphite moderator pieces. It is the estimation of these stresses which is considered in this report for the symmetry condition shown in Figure 1. The stress relief obtained by subdividing the graphite body into four equivalent shapes as indicated in Figure 1 is also considered in this report.

The effect of the fast neutrons upon the graphite structure is to cause an anisotropic straining. The anisotropy is such that the straining in the axial direction (normal to the plane of Figure 1) is different from the straining in the plane of Figure 1 (which is the same in the two planar directions). The amount of straining is proportional to the fast-neutron dose and hence the fast-neutron dose is analogous to the temperature in the rmal-stress theory. F. J. Stanek of Oak Ridge National Laboratory (3) has considered the generalized Hooke's law relating the stresses and strains for the anisotropic situation presented by the graphite stress problem. These stress-strain equations can be written:

(1) References at end of text. 


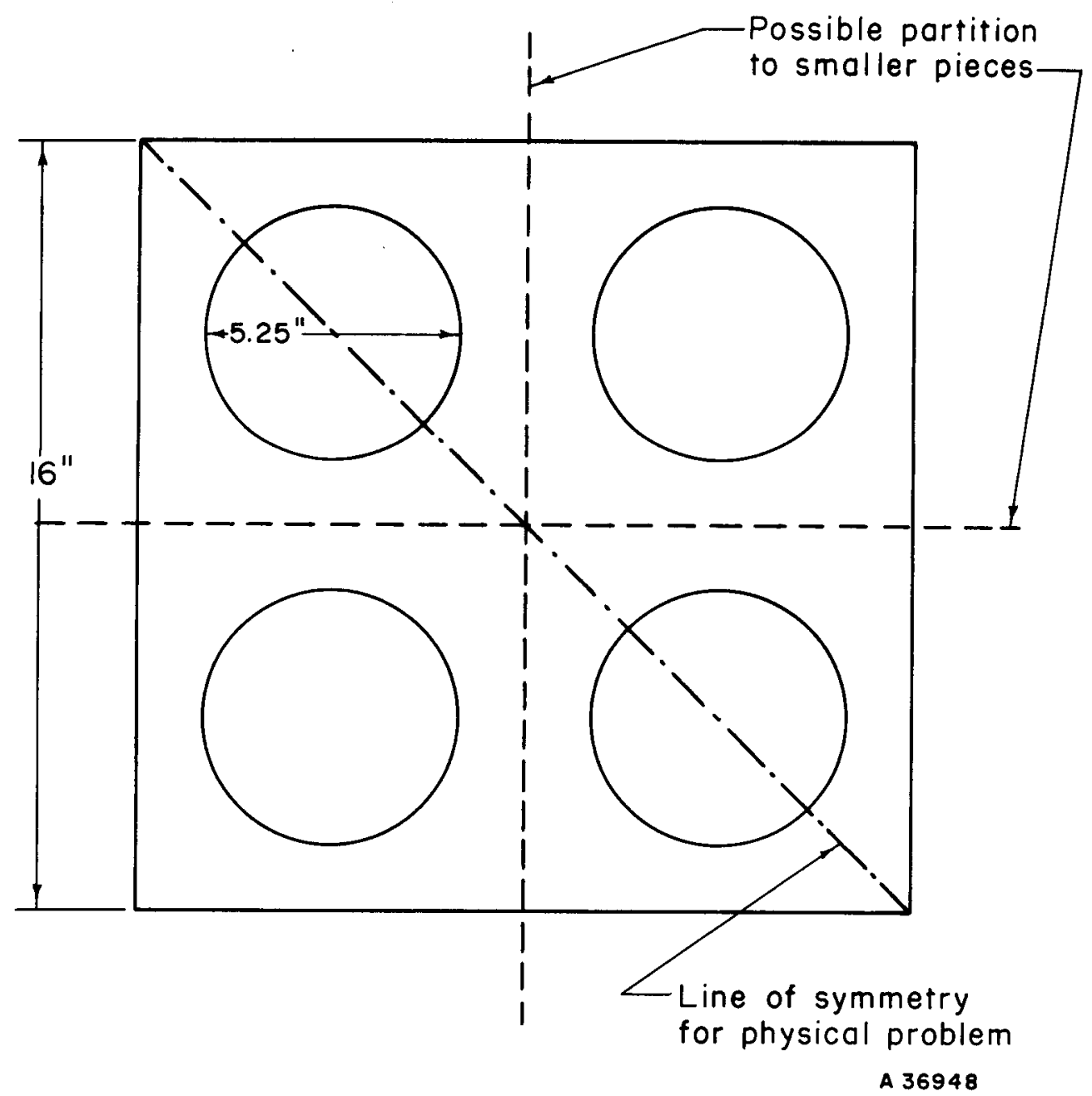

FIGURE 1. MODERATOR ELEMENT FOR THE EXPERIMENTAL GASCOOLED REACTOR (EGCR) 


$$
\begin{gathered}
\epsilon_{x}=\alpha_{x} t+\frac{\sigma_{x}}{E_{x}}-\frac{\delta_{y x}}{E_{y}} \sigma_{y}-\frac{\delta_{z x}}{E_{z}} \sigma_{z}, \\
\epsilon_{y}=\alpha_{y} t-\frac{\delta_{x y}}{E_{x}} \sigma_{x}+\frac{\sigma_{y}}{E_{y}}-\frac{\delta_{x y}}{E_{z}} \sigma_{z}, \\
\epsilon_{z}=\alpha_{z} t-\frac{\delta_{x z}}{E_{x}} \sigma_{x}-\frac{\delta_{y z}}{E_{y}}+\frac{\sigma_{z}}{E_{z}}, \\
\gamma_{x y}=\frac{\tau_{x y}}{G_{x y}}, \gamma_{y z}=\frac{\tau_{y z}}{G_{y z}}, \gamma_{z x}=\frac{\tau_{z x}}{G_{z x}},
\end{gathered}
$$

where the usual terminology(4) for the stresses and strains has been employed and where the subscripts on $\alpha, E$, and $G$ represent the generalization of the Hooke's law constants for anisotropic materials. These constants are however not considered to be independent as:

$$
\frac{\delta_{x y}}{E_{x}}=\frac{\delta_{y x}}{E_{y}}, \frac{\delta_{x z}}{E_{x}}=\frac{\delta_{z x}}{E_{z}}, \frac{\delta_{y z}}{E_{y}}=\frac{\delta_{z y}}{E_{z}},
$$

and consequently it can be shown that:

$$
G_{x y}=G_{y x}=\frac{E_{x}}{1+\frac{E_{x}}{E_{y}}+2 \delta_{x y}}=\frac{E_{y}}{1+\frac{E_{y}}{E_{x}}+2 \delta_{y x}} .
$$

From the simplified anisotropy of the graphite problem it follows that:

$$
\begin{gathered}
E_{x}=E_{y}, G_{z x}=G_{z y} \\
\delta_{x y}=\delta_{y x}, \delta_{x z}=\delta_{y z} .
\end{gathered}
$$

The conditions of plane strain used throughout this report further simplify the problem. These conditions are:

and

$$
\begin{aligned}
& \epsilon_{z}=\text { constant, } \\
& \gamma_{y z}=\gamma_{x z}=0,
\end{aligned}
$$

$$
\int_{A} \sigma_{z} d x d y=0 \text {. }
$$

These conditions imply that the graphite body has no resultant force acting upon it in the $z$-direction; however, resultant moments about the $x$ - and $y$-axes may be required to maintain the plane strain.

For later convenience the following relations are defined: 


$$
\begin{gathered}
\mathrm{X}=\frac{\sigma_{\mathrm{X}}}{\mathrm{D}}, \mathrm{Y}=\frac{\sigma_{\mathrm{Y}}}{\mathrm{D}}, \mathrm{Z}=\frac{\sigma_{\mathrm{z}}}{\mathrm{E}_{\mathrm{z}} \alpha_{\mathrm{z}}}, \\
\mathrm{T}=\frac{\tau_{\mathrm{Xy}}}{\mathrm{D}}, \mathrm{D}=\frac{\mathrm{E}_{\mathrm{z}}\left(\alpha+\beta_{3}\right) \alpha_{\mathrm{z}}}{\beta_{1}-\beta_{3}{ }^{2}}, \alpha=\frac{\alpha_{\mathrm{x}}}{\alpha_{\mathrm{z}}}, \mathrm{E}=\mathrm{E}_{\mathrm{z}}, \\
\beta_{1}=\frac{\mathrm{E}_{\mathrm{z}}}{\mathrm{E}_{\mathrm{X}}}, \beta_{2}=\delta_{\mathrm{Xy}} \frac{\mathrm{E}_{\mathrm{z}}}{\mathrm{E}_{\mathrm{X}}}, \beta_{3}=\delta_{\mathrm{zx}}, \\
\lambda_{1}=\frac{\beta_{3}\left(\alpha+\beta_{3}\right)}{\beta_{1}-\beta_{3}{ }^{2}}, \lambda_{2}=\frac{\beta_{2}+\beta_{3}{ }^{2}}{\beta_{1}-\beta_{3}{ }^{2}}, \lambda_{3}=\frac{\beta_{3}}{\alpha+\beta_{3}},
\end{gathered}
$$

and hence,

$$
\begin{gathered}
\mathrm{Z}=\frac{\epsilon_{\mathrm{Z}}}{\alpha_{\mathrm{z}}}+\lambda_{1}(\mathrm{X}+\mathrm{Y})-\mathrm{t}, \\
\epsilon_{\mathrm{X}}=\left(\alpha+\beta_{3}\right) \alpha_{\mathrm{Z}}\left\{\mathrm{X}-\lambda_{2} \mathrm{Y}+\mathrm{t}-\lambda_{3}\left(\frac{\epsilon_{\mathrm{Z}}}{\alpha_{\mathrm{z}}}\right)\right\}, \\
\epsilon_{\mathrm{y}}=\left(\alpha+\beta_{3}\right) \alpha_{\mathrm{z}}\left\{\mathrm{Y}-\lambda_{2} \mathrm{X}+\mathrm{t}-\lambda_{3}\left(\frac{\epsilon_{\mathrm{z}}}{\alpha_{\mathrm{z}}}\right)\right\}, \\
\gamma_{\mathrm{xy}}=\frac{2\left(\beta_{1}+\beta_{2}\right) \mathrm{D}}{\mathrm{E}} \mathrm{T} .
\end{gathered}
$$

\section{MATHEMATICAL DESCRIPTION}

In this section of the report the equations which describe the plane-strain stress problem are developed. The numerical solutions of these equations are then discussed in the following sections of the report.

In a stress problem besides the stress-strain relations already discussed several additional mathematical requirements must be met. In the first place each element of volume of the body must be in force equilibrium. Considering the $x-$ and $y$-components (the $\mathrm{z}$-component is trivial) there results:

$$
\begin{aligned}
& \frac{\partial X}{\partial x}+\frac{\partial T}{\partial y}=0, \\
& \frac{\partial T}{\partial x}+\frac{\partial Y}{\partial y}=0,
\end{aligned}
$$

if no body forces are acting on the body. It is convenient to introduce at this point the stress function $\phi$ which is related to the stress quantities as follows:

$$
X=\frac{\partial^{2} \phi}{\partial y^{2}}, Y=\frac{\partial^{2} \phi}{\partial y^{2}}, T=-\frac{\partial^{2} \phi}{\partial x \partial y} .
$$


Clearly this definition of the stress function satisfies the requirements of Equation (2) inherently.

A further requirement is introduced because the strains $\epsilon_{x}, \epsilon_{y}$, and $\gamma_{x y}$ are defined in terms of only two displacement functions $u$ and $v$. That is $\epsilon_{x}=\frac{\partial u}{\partial x}, \epsilon_{y}=\frac{\partial v}{\partial y}$, and $\gamma_{x y}=\frac{\partial u}{\partial y}+\frac{\partial v}{\partial x}$ where $u$ and $v$ are $x$ - and $y$-displacements as customarily defined. $(4)$ The so-called compatibility equation is therefore

$$
\frac{\partial^{2} \epsilon_{\mathbf{x}}}{\partial \mathbf{y}^{2}}+\frac{\partial^{2} \epsilon_{\mathbf{y}}}{\partial \mathbf{x}^{2}}=\frac{\partial^{2} \gamma_{\mathbf{x y}}}{\partial \mathbf{x} \partial \mathbf{y}} \text {. }
$$

Equation (4) can be written in terms of the stress function $\phi$ by using Equations (1) and (2):

$$
\nabla^{4} \phi=\nabla^{2} \nabla^{2} \phi=-\nabla^{2} t
$$

Equation (5) is the inhomogeneous biharmonic stress equation.

Another requirement the stress distribution must satisfy is that it must be consistent with the forces applied to the body at the boundary surfaces. If the force components $F_{x}$ and $F_{y}$ at a point on the surface are known and if $n_{x}$ and $n_{y}$ are the components of the outward unit normal vector at the surface point then:

$$
\left\{\begin{array}{l}
F_{x}=n_{x} \sigma_{x}+n_{y} \tau_{x y}, \\
F_{y}=n_{x} \tau_{x y}+n_{y} \sigma_{y} .
\end{array}\right.
$$

For force-free boundary surfaces $F_{x}=F_{y}=0$ and these equations can be written:

$$
\left\{\begin{array}{l}
n_{x} \frac{\partial^{2} \phi}{\partial y^{2}}-n_{y} \frac{\partial^{2} \phi}{\partial x \partial y}=0=F_{x} . \\
-n_{x} \frac{\partial^{2} \phi}{\partial x \partial y}+n_{y} \frac{\partial^{2} \phi}{\partial x^{2}}=0=F_{y} .
\end{array}\right.
$$

These equations are also conveniently written in terms of tangential derivatives at the surface. The unit tangent vector $\vec{s}$ is oriented with respect to the body as shown in Figure 2. Hence

$$
\left\{\begin{array}{c}
-n_{x}=-\frac{d x}{d n}=\frac{d y}{d s}=s_{y}, \\
n_{y}=\frac{d y}{d n}=\frac{d x}{d s}=s_{x},
\end{array}\right.
$$

and

$$
\left\{\begin{array}{c}
F_{x}=-\frac{d}{d s}\left(\frac{\partial \phi}{\partial y}\right)=0, \\
F_{y}=\frac{d}{d s}\left(\frac{\partial \phi}{\partial x}\right)=0 .
\end{array}\right.
$$


Or equivalently,

$$
\begin{aligned}
& \frac{\partial \phi}{\partial y}=\text { constant on surface } \\
& \frac{\partial \phi}{\partial x}=\text { constant on surface } .
\end{aligned}
$$

The boundary equations in the last form are used in the numerical solutions described later.

Finally, the stress solution must be such that the displacements and the rotations are single valued where the body is continuous. The z-component of the rotation is given by

$$
\omega=\omega_{z}=\frac{1}{2}\left(\frac{\partial u}{\partial y}-\frac{\partial v}{\partial x}\right)
$$

This physical requirement does not give any additional equations to be satisfied unless the body is multiconnected (contains holes). If holes are present then this physical requirement must be considered in detail.

The mathematical treatment of this physical requirement of single valuedness follows closely that of Southwell. (5) Consider first the rotation $\omega$. If $\omega$ is to be single valued then

$$
\oint \frac{d w}{d s} d s=0
$$

for any closed path inside the body whether it encloses holes or not. In general, if 1 and 2 represents points within the body then

$$
\omega_{2}=\omega_{1}+\int_{1}^{2} \frac{d w}{d s} d s,
$$

where the path of integration between 1 and 2 is arbitrary as long as it is inside the body. Thus,

$$
\begin{aligned}
\omega_{2} & =\omega_{1}+\int_{1}^{2}\left[\frac{d x}{d s} \frac{\partial \omega}{\partial x}+\frac{d y}{d s} \frac{\partial \omega}{\partial y}\right] d s \\
& =\omega_{1}-\int_{1}^{2}\left[\frac{d y}{d n}\left(\frac{\partial \epsilon_{x}}{\partial y}-\frac{1}{2} \frac{\partial \gamma_{x y}}{\partial x}\right)\right. \\
& \left.+\frac{d x}{d n}\left(\frac{\partial \epsilon_{y}}{\partial y}-\frac{1}{2} \frac{\partial \gamma_{x y}}{\partial y}\right)\right] d s,
\end{aligned}
$$

but

$$
\epsilon_{x}=\epsilon\left[\frac{\partial^{2} \phi}{\partial y^{2}}-\lambda_{2} \frac{\partial^{2} \phi}{\partial x^{2}}+t-\lambda_{3} \bar{t}\right]
$$




$$
\begin{aligned}
& \epsilon_{y}=\epsilon\left[\frac{\partial^{2} \phi}{\partial x^{2}}-\lambda 2 \frac{\partial^{2} \phi}{\partial y^{2}}+t-\lambda_{3} \bar{t}\right] \\
& \gamma_{x y}=-2 \gamma \frac{\partial^{2} \phi}{\partial x \partial y}, \gamma=\frac{\left(\beta_{1}+\beta_{2}\right) D}{E} \\
& \epsilon=\left(\alpha+\beta_{3}\right) \alpha_{z}, \bar{t}=\int_{A} \frac{t d x d y}{\int_{A} d x d y} .
\end{aligned}
$$

The term $\bar{t}$ in $\epsilon_{x}$ and $\epsilon_{y}$ results from the condition $\int \sigma_{z} d x d y=0$ and an application of Green's theorem using the boundary conditions, Equation (10).

Finally these results after some rearrangement

$$
\omega_{2}=\omega_{1}-\epsilon \int_{1}^{2} \frac{d}{d n}\left(\nabla^{2} \phi+t\right) d s,
$$

which in the case of a closed path becomes

$$
\oint \frac{d}{d n}\left(\nabla^{2} \phi+t\right) d s=0 .
$$

In a similar fashion the displacements $u$ and $v$ can be treated to obtain

$$
\begin{array}{r}
\left.\left.\right|_{1} ^{u}\right|_{1} ^{2}=\left|-y \omega-\gamma \frac{\partial \phi}{\partial x}-\epsilon \lambda_{3} x \bar{t}\right|_{1}^{2} \\
+\epsilon \int_{1}^{2}\left(-y \frac{d}{d n}+\frac{d x}{d s}\right)\left(\nabla^{2} \phi+t\right) d s,
\end{array}
$$

and

$$
\begin{gathered}
\left.\left.\right|_{v}\right|_{1} ^{2}=\left|x \omega-\gamma \frac{\partial \phi}{\partial y}-\epsilon \lambda_{3} y \bar{t}\right|_{1}^{2} \\
+\epsilon \int_{1}^{2}\left(x \frac{d}{d n}+\frac{d y}{d s}\right)\left(\nabla^{2} \phi+t\right) d s .
\end{gathered}
$$

Again for a closed path

$$
\oint\left(y \frac{d}{d n}-n_{y}\right)\left(\nabla^{2} \phi+t\right) d s=0
$$

and

$$
\oint\left(x \frac{d}{d n}-n_{x}\right)\left(\nabla^{2} \phi+t\right) d s=0
$$

Since the closed path may include only one hole and can be deformed to coincide with the contour of the hole, the requirement that $\omega, u$, and $v$ be single valued everywhere in the body can be satisfied if Equations (12), (15), and (16) are satisfied for each hole 
contour. These equations must therefore be considered if the body contains holes. These equations are satisfied trivially if the path does not enclose a hole as can be seen by applying Green's theorem to $\nabla^{4} \phi, x \nabla^{4} \phi$, and $y \nabla^{4} \phi$ in turn.

Thus a solution to the stress problem must satisfy Equation (5) for every interior point, Equation (10) for every boundary point, and Equations (12), (15), and (16) for every hole contour. This represents the mathematical statement of the problem.

\section{NUMERICAL DESCRIPTION}

In this section of the report the differential equations are approximated by linear algebraic equations which can then be solved numerically. The program for accomplishing this is to select a finite number of points in the body at which the stress function $\phi$ is to be evaluated. In order to solve the differential stress equations in some approximate sense, relations between the stress function at neighboring points are introduced. Finally a simultaneous set of linear algebraic equations is obtained equal in number to the number of points at which the stress function is to be specified. The solution of the algebraic system then furnishes an approximate solution for the stress function.

A problem of fundamental importance in this program is that of representing a continuous function by a formula involving only a finite number of discrete point values of the function. This is nothing more however than the interpolation problem in numerical analysis. A systematic approach in making the transition from differential equations to algebraic equations makes use of appropriate interpolation formulas. Thus consider the two dimensional array of points shown in Figure 3. This array is sometimes termed the biharmonic array. It may be asked to find a polynomial expression in $\mathrm{x}$ and $\mathrm{y}$ which assumes the correct value of some arbitrary function at each of the 13 points in the array. One such polynomial expression is obtained by a double application of Stirling's interpolation formula. (6) The result is as follows:

$$
\begin{aligned}
\phi(r, s)=\phi(7) & +\frac{r}{2}[\phi(8)-\phi(6)]+\frac{s}{2}[\phi(3)-\phi(11)]+\frac{r^{2}}{2}[\phi(8)+\phi(6)-2 \phi(7)] \\
& +\frac{s^{2}}{2}[\phi(8)+\phi(11)-2 \phi(7)]+\frac{r s}{4}[\phi(4)+\phi(10)-\phi(2)-\phi(12)] \\
& +\frac{r\left(r^{2}-1\right)}{12}[\phi(9)-2 \phi(8)-\phi(5)+2 \phi(6)]+\frac{s\left(s^{2}-1\right)}{12}[\phi(1)-2 \phi(3)-\phi(13)+2 \phi(11)] \\
& +\frac{r s^{2}}{4}[\phi(4)+\phi(12)-2 \phi(8)-\phi(10)-\phi(2)+2 \phi(6)] \\
& +\frac{r^{2} s}{4}[\phi(4)+\phi(2)-2 \phi(3)-\phi(12)-\phi(10)+2 \phi(11)] \\
& +\frac{r^{2}\left(r^{2}-1\right)}{24}[6 \phi(7)-4 \phi(8)-4 \phi(6)+\phi(9)+\phi(5)]
\end{aligned}
$$




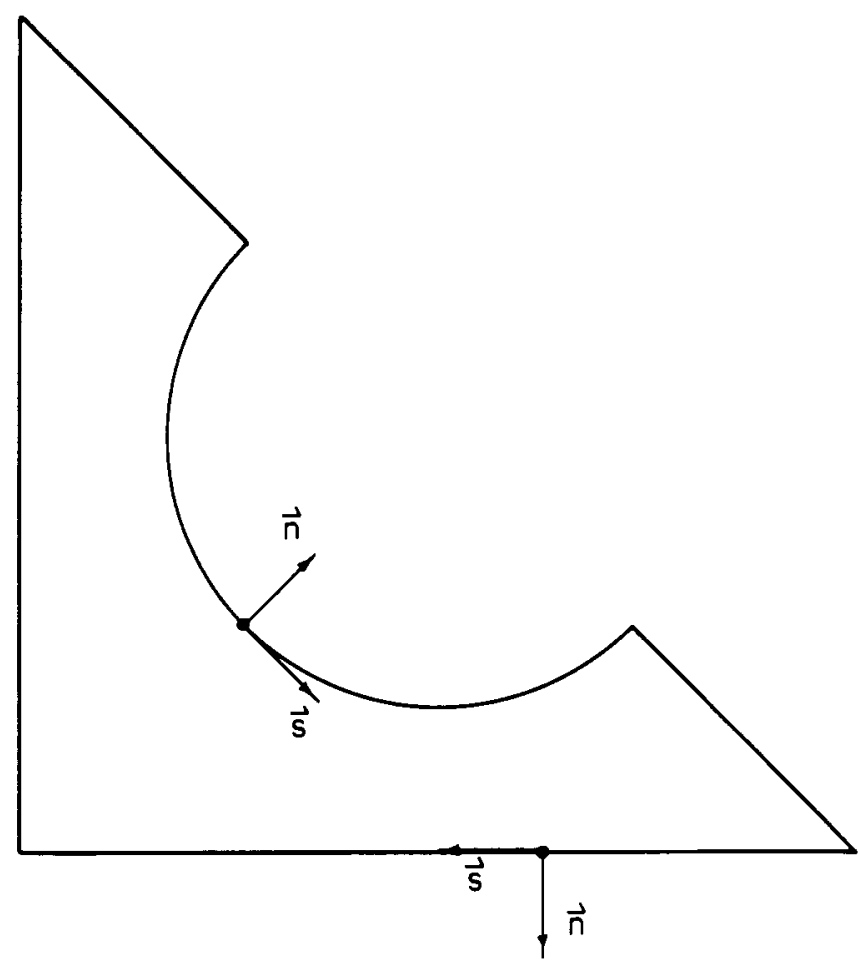

FIGURE 2. ORIENTATION OF UNIT VECTORS AT BOUNDARY POINTS

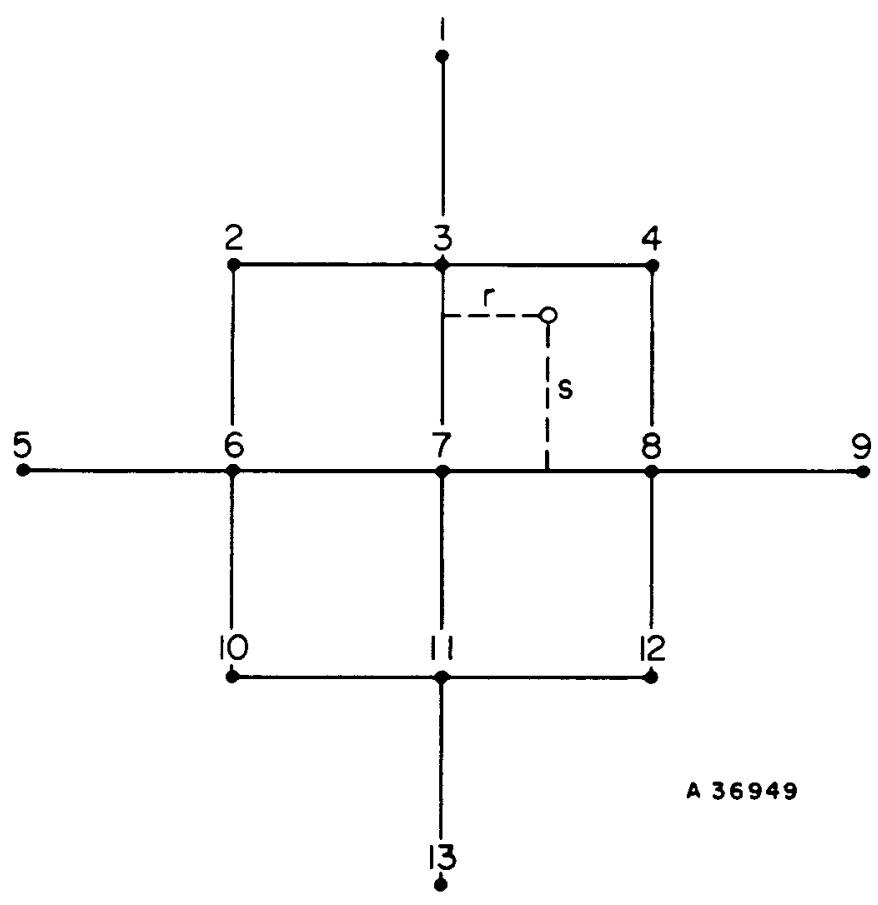

FIGURE 3. BIHARMONIC ARRAY OF MESH POINTS WITH ( $x-s$ ) EXAMPLE FOR THE INTERPOLATION FORMULA 


$$
\begin{aligned}
& +\frac{s^{2}\left(s^{2}-1\right)}{24}[6 \phi(7)-4 \phi(3)-4 \phi(11)+\phi(1)+\phi(13)] \\
& +\frac{r^{2} s^{2}}{4}[\phi(4)+\phi(2)+\phi(12)+\phi(10)-2 \phi(3)-2 \phi(8)-2 \phi(6)-2 \phi(11)+4 \phi(7)] .
\end{aligned}
$$

It may be verified that $\phi(r, s)$ attains its proper value at each of the 13 points in the biharmonic array. Equation (17) may then be differentiated and evaluated at some appropriate point to obtain an algebraic equation involving the 13 point values of $\phi, \phi(1)$, $\phi(2)$, etc. As an example, if the biharmonic operation is applied Equation (17) for $\phi$ $(r, s)$ there results

$$
\begin{gathered}
\nabla^{4} \phi(\mathrm{r}, \mathrm{s})=20 \phi(7)-8[\phi(3)+\phi(6)+\phi(8)+\phi(11)]+2[\phi(2)+\phi(4)+\phi(10)+\phi(12)] \\
+[\phi(1)+\phi(5)+\phi(9)+\phi(13)],
\end{gathered}
$$

an expression which does not involve $r$ and $s$.

In this way the stress equations, Equations (5), (10), (12), (15), and (16), can be approximated by algebraic equations. Of course the integral conditions, (12), (15), and (16) must also involve numerical integrations but more will be said of this later.

Now the selection of the finite set of points to evaluate the stress function will be discussed. Consider a body of cross-sectional shape as shown in Figure 4a. This is one quadrant of the shape shown in Figure 1 and can be related to the stress problem when the body in Figure 1 is subdivided. Suppose that the neutron dose function $t$ is symmetrical about one diagonal. Figure $4 \mathrm{~b}$ shows the symmetry element and also shows a superposed mesh. The mesh points which are shown as blackened circles (interior points) are in the interior or on the boundary of the symmetry shape. The mesh points which are shown as open circles (exterior points) are exterior to the shape but are in the biharmonic array of some interior point. These points, exterior and interior, are then the points at which the stress function is to be evaluated. The exterior points generally have no physical significance. Mathematically they can be considered as representing an analytic continuation of the stress function beyond the physical boundary. The exterior points in the image portion of the symmetry shape, of course, do have some physical significance.

There are several reasons for taking the exterior points into consideration. First, the boundary equations are somewhat better represented by central difference interpolation formulas. Second, the most severe stress conditions frequently exist on the boundary and hence it is desirable to be able to compute the stresses at the boundary with some accuracy. The stresses involve differentiations of the stress function, and this emphasizes the importance of having a good numerical representation of the stress function near the boundaries.

The next step is the selection of the equations equal in number to the number of mesh points selected. To each interior point can be associated a biharmonic equation, Equation (5) (t specified) using Equation (18), with Point 7 selected to coincide with the interior point. The boundary equations can be approximated by selecting points on the boundaries, not necessarily mesh points, and writing the appropriate algebraic expressions at the se points. It has been customary in the work reported here to space 

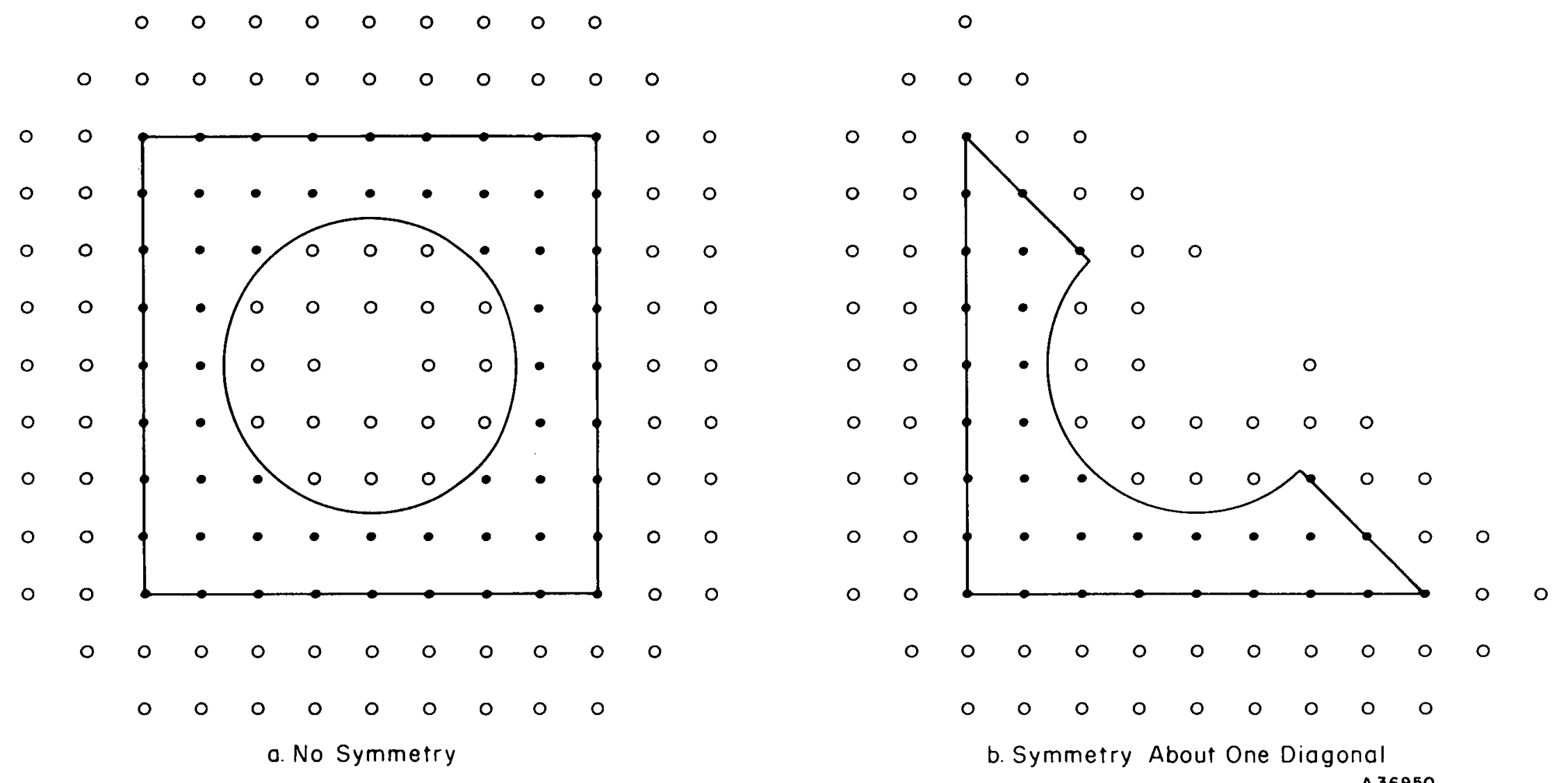

FIGURE 4. SHAPES CONSIDERED FROM PARTITIONING WITH SAMPLE MESH-POINT ARRAYS (THESE WERE NOT USED) 
boundary points equidistantly along each boundary curve. The integral conditions, Equations (12), (15), and (16), are then written algebraically by performing a trapezoidal rule integration along the appropriate boundary using the boundary points to write the point values for the integrands. A boundary point is specified by giving its $r$, s relationship to a specified interior point nearby.

The above procedures are then applied to obtain a set of independent linear algebraic equations which determine the stress function uniquely at the mesh points selected. Of course the selection of the equations is somewhat arbitrary and hence the resulting stress-function solution will vary to some extent according to this selection, but this is a problem with any approximate numerical scheme.

A unique solution to the system of equations is required in order to solve the system numerically. Since the stress problem is arbitrary to a constant in the stress function, the value of the stress function can be specified at some arbitrary value at some arbitrary mesh point. It has been customary to specify the stress function to be zero as some selected exterior point. In addition, in most problems the stress function is arbitrary to the extent that a term $a x+b y$ with $a$ and $b$ arbitrary can be added. This arbitrariness can be eliminated by choosing the constants in Equation (10) both to be zero on the external boundary of the body. It should be noted though that the constants on the hole boundaries are not so restricted. Of course, some care must also be exercised so that redundant equations are not written.

Once the stress-function values at the mesh points have been determined by numerical solution the $x y$-phase stresses and the displacements can be determined numerically by the use of Equations (3), (11), (13), (14), and (17) in the appropriate manner. Since the displacements involve line integrations it has been customary to calculate only the relative displacements along boundaries.

The axial stress can be obtained from the expression

$$
\frac{\sigma_{Z}}{E \alpha_{Z}}=Z=\lambda_{1}(X+Y)-(t-\bar{t}) \text {. }
$$

The bending moments about the $\mathrm{x}$ - and $\mathrm{y}$-axes can then be obtained by integration

$$
\begin{aligned}
& M_{x}=\iint_{A} \sigma_{z} y d x d y . \\
& M_{y}=\iint_{A} \sigma_{z} x d x d y
\end{aligned}
$$

In the area integrations involved in Equation (20) and in the $\bar{t}$ a very approximate value is obtained by averaging the integrand over the interior mesh-point distribution. This approximation is used in the work reported here.

\section{NUMERICAL SOLUTION}

As already indicated the numerical description of the thermal-stress problem leads to the problem of solving a set of simultaneous linear algebraic equations for the stress-function values at the mesh points. The number of equations in the system may 
be quite large. Systems as large as a 1000 have been considered in the work reported here. For such large systems direct solution methods are impractical and iterative methods become attractive. An example of an interative method is the relaxation method. (4) Modern digital computers, however, can more readily solve sequential methods of iteration.

An iterative procedure has been described previously(1) which retains some of the features of a relaxation method but which is sequential and can be readily programmed for machine computation. This method is also described in Appendix A. As with most iterative methods for solving the biharmonic equations the convergence rate is poor and hence a good initial guess is necessary. The procedure followed here is to solve a coarse-mesh problem by a direct method (Gaussian elimination). This coarse-mesh solution is then used to interpolate an initial guess for a mesh spacing half as large which is then iterated. Another interpolation is then made for an initial guess for a mesh spacing again half as large. This is then iterated to obtain the final approximation solution for the doubly refined mesh. The stresses, displacements, and bending moments are then computed from the approximate solution for the stress function.

As indicated in Appendix $A$ there is some choice to be made in the extrapolation factor and in the frequency of block extrapolation in the iteration. These choices have somewhat arbitrarily been made so that the extrapolation factor is unity and block extrapolation is performed every ten iteration cycles. Very little is known at this point about the behavior of the iteration convergence for different choices of these parameters.

As described in Appendix A the iteration scheme solves the system

$$
\begin{aligned}
& A_{11} \phi_{1}+A_{12} \phi_{2}+\cdots+A_{1 R} \phi_{R}=21 \\
& A_{21} \phi_{1}+A_{22} \phi_{2}+\cdots+A_{2 R} \phi_{R}=a_{2} \\
& A_{R 1} \phi_{1}+A_{R 2} \phi_{2}+\cdots+A_{R R} \phi_{R}=a_{R}
\end{aligned}
$$

or $A \phi=a$ in matrix form, by reducing the length of the residual vector at each step, i.e., $\mathrm{r}^{2}=|\mathrm{A} \phi-\mathrm{a}|^{2}$ is made smaller at each step. This is analogous to the strategy of relaxation although in the latter the place in the mesh where the reduction is made is a matter of choice. The convergence of the iteration scheme is somewhat similar to what is observed in a relaxation solution: the residuals can be reduced quite significantly at the early stages of the iteration but less and less so as the iteration proceeds. Of course this is characteristic of almost any iteration process. The method of iteration used, like relaxation, has the property of giving a smooth solution approximation. This is particularly advantageous in the stress problem since the stress function must be differentiated twice to yield the stress components.

\section{COMPUTER CODES}

The numerical procedures already described were coded in FORTRAN. The numerical calculations were then performed on the IBM-7090. The codes were developed specifically for a $32 \mathrm{~K}$ machine. In this section of the report the computer codes will be described in general terms whereas the details will be found in Appendix $B$. 
In order to make the codes as flexible as possible in application it is necessary to use a large amount of input information. A part of this input is just a matter of constructing tables of integers which describe the way in which equations are assigned to boundary points. On the other hand, a part of the input describes the normal vector and the $r$, s quantities for every boundary point, and this involves some preliminary computation, in general.

\section{Matrix-Calculation Code (I)}

This part of the report describes the calculation of the coefficient matrix for the stress problem. Several options are available for the right hand side of the biharmonic equation to be solved. These options are selected by specifying the appropriate value of IQC $(=1,2,3$, or 4$)$ as part of the input. Thus,

$$
\nabla^{4} \phi=\left\{\begin{array}{l}
0 \text { if IQC }=1 \\
I \text { if IQC }=2 \\
f(x, y) \text { if } \text { IQC }=3 \\
-B^{2} t(x, y) \text { if IQC }=4 .
\end{array}\right.
$$

The last option is the one used in the numerical work described here. This option follows from $\nabla 2_{t}=B 2_{t}$ since the "temperature" function $t$ is proportional to the fastneutron flux $\phi_{\mathrm{f}}$ which satisfies $\nabla^{2} \phi_{\mathrm{f}}=\mathrm{B}^{2} \phi_{\mathrm{f}}$ in the graphite region under study. In these options $f(x, y)$ or $t(x, y)$ are specified at each interior mesh point as part of the input.

The input preparation for a selected mesh is now described. Figure 5 shows the system to be considered. The detailed input for this problem is described in Appendix C. A rectangle is then constructed which just includes all of the mesh points. The mesh points are then numbered sequentially as shown in Figure 5. The points of the rectangular array are also numbered sequentially. Then a table of correspondence is prepared. This table, the ITABC $\emptyset$ (I) table, lists in sequence the sequence number of the rectanular array points which coincide with the mesh points. Thus $I E=87$ is the number of mesh points and the ITABC $\emptyset$ table has IE entries. Figure 5 shows an example: $\operatorname{ITABC} \emptyset(9)=29$.

The boundary points are then specified as described in this report under "Numerical Description". A boundary curve is defined for each portion of the boundary which is between corners or is closed and is assigned a boundary-curve number, say I. Thus $I=1$ for the horizontal edge, $I=2$ for the vertical edge, and $I=3$ for the hole segment. The diagonal edge is not defined as a boundary curve here because symmetry equations can be used for the image points without introducing boundary points. However, if a symmetry line does not have the 45-deg character and does not lie on the mesh points, the symmetry line must also be given a curve number and appropriate boundary points.

The boundary points are then selected at equal intervals on each curve although the interval size can differ from curve to curve. Each boundary point is then assigned a sequence number, starting with Curve $l$ and continuing through the curve sequence. The first and last boundary point on each curve is given a unique sequence number even though such a point may have as a result two sequence numbers. For example, the last point on Curve 1 is the first point on Curve 2 when the numbering starts at the lower 


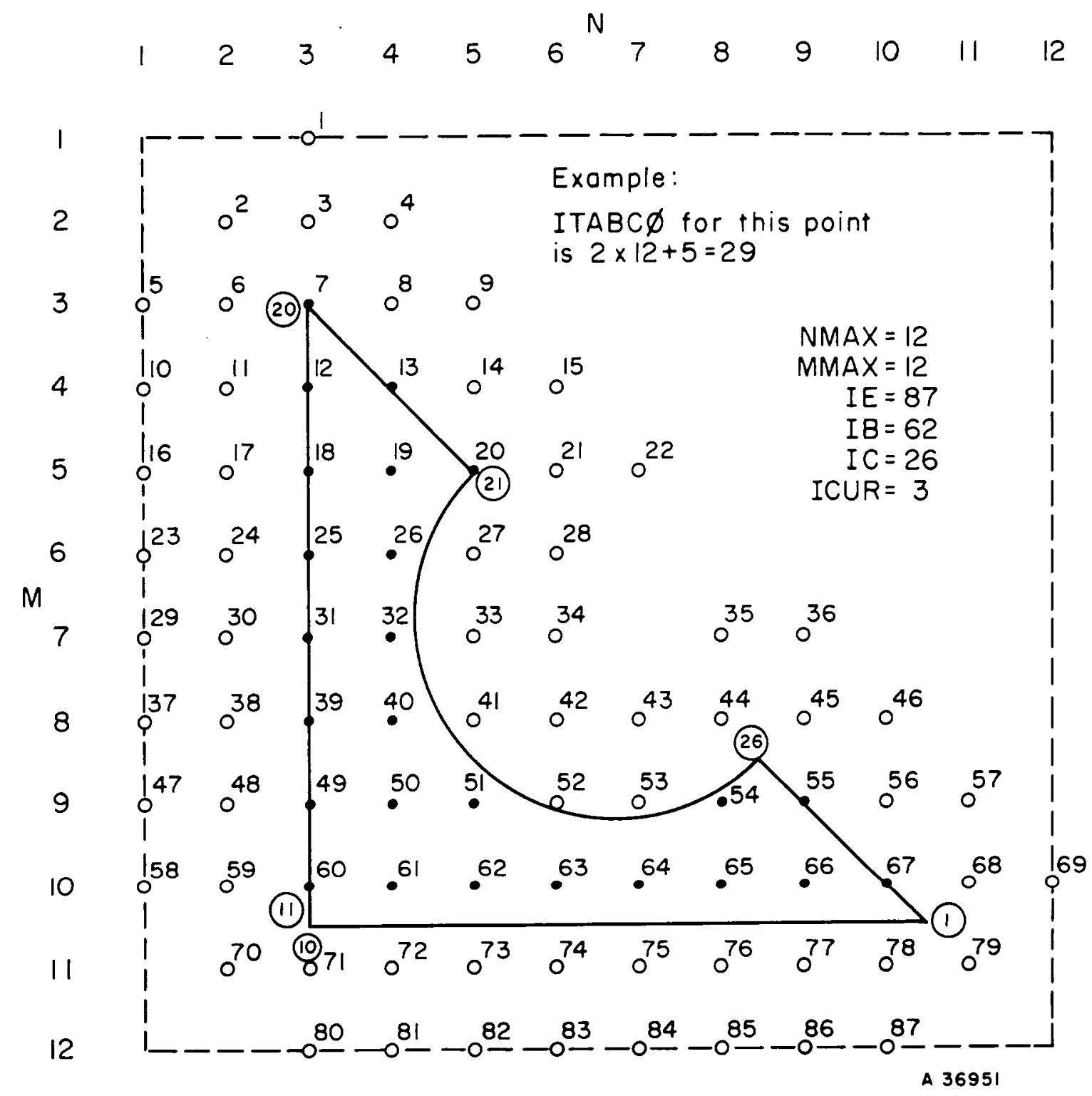

FIGURE 5. MESH-POINT ARRAY USED FOR SHAPE IN FIGURE $4 a$

Boundary points at corners are shown as circled numbers. 
right corner and proceeds clockwise. The number of boundary curves selected is denoted by ICUR and the first and last points on each boundary are denoted by IBEG (I) and IEND (I) for $I=1$, ICUR.

For each boundary point it is necessary to specify: (1) the outward normal vector components $N X$ and $N Y$ (for $n_{x}$ and $n_{y}$ ), (2) the interior mesh point which is to be the center for the interpolation formula, and (3) the $r$, s relationship of the boundary point to this interior point. The $r$, s values are denoted by $R, S$. The interior mesh point of (2) is designated by specifying the row (M) and the column (N) indices for this point in the rectangular array where $1 \leqslant M \leqslant M M A X, 1 \leqslant N \leqslant N M A X$. Thus the rectangular array already discussed has MMAX rows and NHAX columns. The row $M=1$ is the top row and the column $N=1$ is the left-most column. The boundary-point information is prepared as input in the form of Tables NX(I), NY(I), R(I), S(I), M(I), N(I), where I = $1, I C$ where $I C$ is the number of boundary-point sequence numbers.

There remains the problem of associating a nonbiharmonic equation with each exterior mesh point. This association is prepared as input by specifying tables with IB entries where IB is the number of exterior mesh points. First, the IPMSN(I) table gives in sequence the mesh-point sequence numbers of the exterior points. Second, the ICN(I) table specifies an equation-type number for each exterior point. Third, the IBDSN(I) table gives the boundary point appropriate to the equation type for each exterior point. Third, the IEXPNS(I) table gives the center point for the interpolation formula appropriate to the boundary-point assignment.

The ICN(I) table as mentioned gives the equation-type number. These are described in detail in Appendix B. However, certain rules must be followed in preparing the input. If $I C N(I)$ is 9,10 , or 11 the IBDSN entry for this I must be the IBEG number for this curve. On the other hand, if ICN(I) is 7 or 8 the IBDSN entry for this I must not be the IBEG for any curve. The reason for the first restriction is that the integrations are started at the boundary point indicated by the IBDSN number. The reason for the second restriction is that the boundary point at the beginning of each curve is taken as the reference point for specifying the value of the derivative, and a redundant equation will result if this restriction is not observed.

Several additional features must be noted. If a symmetry condition, Equation (12), can be employed, the mesh point which is the image of the IPMSN(I) must be entered as IEXPNS(I). If a symmetry line cuts through a hole, the angle must be specified which is required to rotate the symmetry line into the $y$-axis in a clockwise direction. This angle must be specified in radians and is denoted by THET(I) where I = 1, ICUR. If I denotes a curve which is not cut by a symmetry line then THET(I) must be entered as zero. If a single symmetry line cuts through a hole an Equation Type 10 must not be written for that hole boundary as it will be a redundant equation but Equation Types 9 and 11 must be written for that hole boundary. If two symmetry lines cut a hole then Equation Types 10 and 11 must not be written and Equation Type 9 must be written for this hole boundary. In this last case THET(I) is also entered as zero.

Also to be noted is that the biharmonic equation, Type 1 , will be written automatically since any mesh point not in the IPMSN table is assumed to be an interior point and Equation Type $l$ is automatically written for each interior point. The point at which the stress function is set equal to zero must therefore be an exterior point. Hence, for some I, ICN(I) must be 2 . 
The preceding discussion covers most of the input required to construct the matrix elements. The "temperature" function $t$ must, of course, be specified for each mesh point. This is done by means of the TEMP(I) table where $I=1, \ldots$, IE. However, a few comments are pertinent. The temperature function is required only at the interior points and those exterior points which are in a Laplacian array (Points 3, 6, 7, 8, and 11 of Figure 3) about some interior point as center. Further, of the exterior point values only those required for the integral-type equations, Types 9,10 , and 11 , are needed. Those temperature values not needed as indicated may be entered as zero in the TEMP table. The Laplacian array is used for the temperature function because in many applications this array is the one used to determine the temperature function numerically rather than the biharmonic array.

The coefficients in each equation are normalized so that the sum of the coefficients squared is unity in each equation. This scaling is done to give comparable weighting to each equation in the iteration scheme.

\section{Matrix-Transpose Code (II)}

The transpose of the coefficient matrix is used extensively in the iteration routine (see Appendix A). The matrix-transpose code obtains the transposed matrix from the matrix and prepares it for input to the iteration routine. The input and the restriction are given in Appendix B. However, it should be noted that this code will function most efficiently if $\mathrm{N} \emptyset \mathrm{NZ}$ is made as small as possible

\section{Iteration Code (III)}

The matrix calculation code already discussed calculates from the input the coefficient matrix, $A$, and the right-hand-side vector, a, for the stress problem [see Equation (21)]. This information is then used with the transposed matrix and other input to carry out the iterative solution described in Appendix A.

The additional inputs required are an initial guess for the solution vector, $\phi$, and values for parameters which enter into the iteration scheme. The initial-guess-vector is obtained from an interpolation of the solution vector for a mesh size twice the size being considered. The solution vector for the coarsest mesh is obtained by a direct method (Gaussian elimination).

The parameters for the iteration code are defined in Appendix B. The parameter $\alpha$ has been taken as 1.0 in the numerical work. N3 has been taken as 300,500, and 1000 for various problems. N4, the number of iteration cycles between block extrapolation, has usually been taken as 10. N5 has usually been taken so that a regeneration is performed only at the end of the iteration. It has been found that round-off error accumulation is not important even after a thousand cycles. The iteration stop involving $X$ has not been used and $X$ is set equal to zero.

The behavior of the convergence of the iteration scheme for different choices of $\alpha$, N4, and the initial guess has not as yet been explored. Usually for the choices of the parameters which have been made the iteration does not accomplish much after several hundred iterations. The $\mathrm{K}$ factor for the block extrapolation has been observed to fall 
generally in the range of from ten to several hundred for the parameter choices indicated above. Some typical results are indicated in Table 1 for two-mesh spacings for the problem corresponding to Figure 5.

\section{TABLE 1. TYPICAL COMPARISON OF NUMERICAL RESULTS FOR DIFFERENT MESH SIZES}

\begin{tabular}{lll}
\hline \multicolumn{1}{c}{$\begin{array}{c}\text { Residual Vector } \\
\text { Squared and Block } \\
\text { Extrapolation Factor }\end{array}$} & $\begin{array}{c}\text { Intermediate } \\
\text { Mesh } \\
\mathrm{IE}=211\end{array}$ & $\begin{array}{c}\text { Fine } \\
\text { Mesh } \\
\mathrm{IE}=563\end{array}$ \\
\hline $\mathrm{r}^{2}$ (residual vector squared) initially & $3.1 \times 10^{-1}$ & $8.6 \times 10^{-2}$ \\
$\mathrm{r}^{2}$ after 250 interactions & & $2.7 \times 10^{-5}$ \\
$\mathrm{~K}$ after 250 iterations & & 31 \\
$\mathrm{r}^{2}$ after 500 iterations & $1.4 \times 10^{-4}$ & $1.8 \times 10^{-5}$ \\
$\mathrm{~K}$ after 500 interations & 166 & 16 \\
$\mathrm{r}^{2}$ after 1000 iterations & $5.9 \times 10^{-5}$ & \\
$\mathrm{~K}$ after 1000 iterations & 103 & \\
\hline
\end{tabular}

The slowing down of the convergence rate is apparent from Table 1.

\section{Stress-Computation Code (IV)}

After the stress function has been determined either by a direct solution or by the iterative solution, the stresses, moments, and displacements can be calculated. The calculation of the stresses and the bending moments is done by the routine now being discussed. The displacement calculation routine will be discussed in the following section.

This routine calculates $\sigma_{x}, \sigma_{y}$, and $\tau_{x y}$ for each interior mesh point. In addition, the principal stresses are computed and the maximum shear stress is computed. The $\sigma_{z}$ component is calculated as $\sigma_{z}\left(E_{z} \alpha_{z} / D\right)$ for each interior point for each choice of $\lambda_{1}$ [see Equation (19)] for up to five choices of $\lambda_{1}$ which are denoted by NU $Z(I), I=1, \ldots$, 5. The points in the mesh where the limiting stresses are attained are also selected. Finally the bending moments are calculated as discussed previously.

\section{Displacement-Computation Code (V)}

The rotation and displacements are calculated from the stress function according to Equations (11), (13), and (14) for each boundary curve. For each curve the rotation and displacements are calculated at each boundary point relative to the initial boundary point for the curve. The input-output details are given in Appendix B. 
Interpolation Code (VI)

This code takes the stress function for a given mesh and interpolates according to Equation (17) to find the stress function at the mesh points for a mesh spacing half as large. Thus the solution vector for a given mesh can be used to find the initial guess vector for a halved mesh automatically which is in a form to use directly in the iteration routine. Again the input-output details are given in Appendix B. However, several new tables are needed as input to the Interpolation Code. There are IE mesh points in the starting mesh and IFE mesh points in the refined mesh. The ITABFM, IEXPAN, and ISM tables each have IFE entries and each table sequence follows that of the IFE mesh points of the refined mesh. The entries of the ITABFM tables are the ITABC $\emptyset$ entries for the fine mesh (see ITABC $\emptyset$ table in Appendix B). The IEXPAN entries are such that a zero is entered if the refined mesh point coincides with a point of the initial mesh, otherwise the mesh-point number in the refined mesh sequence of a nearby interior point of the initial mesh is entered. This interior point is then used as the center of the biharmonic array used in the interpolation formula. If the IEXPAN entry is zero, the corresponding ISM entry is not zero and conversely. The ISM entry in the nonzero case is the mesh-point number in the initial mesh sequence which coincides with the mesh point in the refined mesh under study.

GEL 1 Input Code (VII)

As discussed earlier the solution vector for the coarsest mesh is obtained by Gaussian elimination. The code used is NYGEL $1(7)$, which is a SHARE subroutine. The Gel 1 Input Code prepares the coefficient matrix from Code I in a format acceptable to NY GEL 1. The input-output details are given in Appendix B.

\section{COMBINING CODES}

As indicated in Appendix B, it is possible to perform the computations with the preceding codes sequentially on the computer. This requires, however, a large amount of error-free input data. As a rough indication of the computing time required for a typical problem (IBM-7090), Table 2 shows the running times for various stages of the complete calculation for the three mesh sizes considered for the problem relating to Figure 5. 
TABLE 2. TYPICAL RUNNING TIMES FOR PROGRAM SEQUENCE

\begin{tabular}{|c|c|c|}
\hline $\begin{array}{l}\text { Running } \\
\text { Time, } \\
\text { min }\end{array}$ & \multicolumn{2}{|l|}{ Routine } \\
\hline 1 & 1. Coefficient matrix & $I E=87$ \\
\hline 15 & 2. GEL 1 & "coarse" mesh \\
\hline 1 & 3. Stress Computation & \\
\hline 1 & 4. Interpolation & \\
\hline 2 & 5. Coefficient matrix & \\
\hline 2 & 6. Matrix transpose & $\begin{array}{l}1 \mathrm{E}=211 \\
\text { "intermedia }\end{array}$ \\
\hline 20 & 7. Iteration ( 1000 Cycles) & "intermediate" \\
\hline 1 & 8. Stress Computation & mesh \\
\hline 2 & 9. Interpolation & \\
\hline 3 & 10. Coefficient matrix & \\
\hline 5 & 11. Matrix transpose & \\
\hline 20 & 12. Iteration $(500$ cycles) & $I E=536$ \\
\hline 1 & 13. Stress computation & "fine" mesh \\
\hline 1 & 14. Displacement computation & \\
\hline
\end{tabular}

\section{DISCUSSION OF RESULTS}

Numerical solutions were obtained for the moderator piece shown in Figure 1 for a "temperature" function symmetric about one diagonal as shown also in Figure 1. The full-size element was considered and the one-quarter-size elements obtained by subdivision were also considered. Figure 6 shows the geometrical shapes considered after the symmetry was included.

The "temperature" function, $t$, was known from numerical results obtained by Oak Ridge National Laboratory. The "temperature" function is proportional to the total exposure to fast neutrons. Since the holes contain the sources of the fast neutrons, the temperature function peaks at the hole boundaries. Figure 7 shows the behavior of the "temperature" function along the symmetry diagonal shown in Figure 1. It may be noted that an increase of the "temperature" tends to make the graphite shrink.

For the specified "temperature" function, the shape of Figure 6a was studied for two mesh spacings. The coarse mesh spacing involved 231 equations and the refinement of this mesh involved 602 equations. An initial guess for the stress function was obtained by solving the problem with symmetry about both diagonals for the coarse mesh spacing (138 equations). Points $A$ and $B$ in Figure $6 a$ show where the maximum tensile stresses in the $x y$-plane were found (considering only the stresses evaluated at mesh points). Table 3 shows the value of the maximum tensile stresses at these points for a chosen exposure condition. 


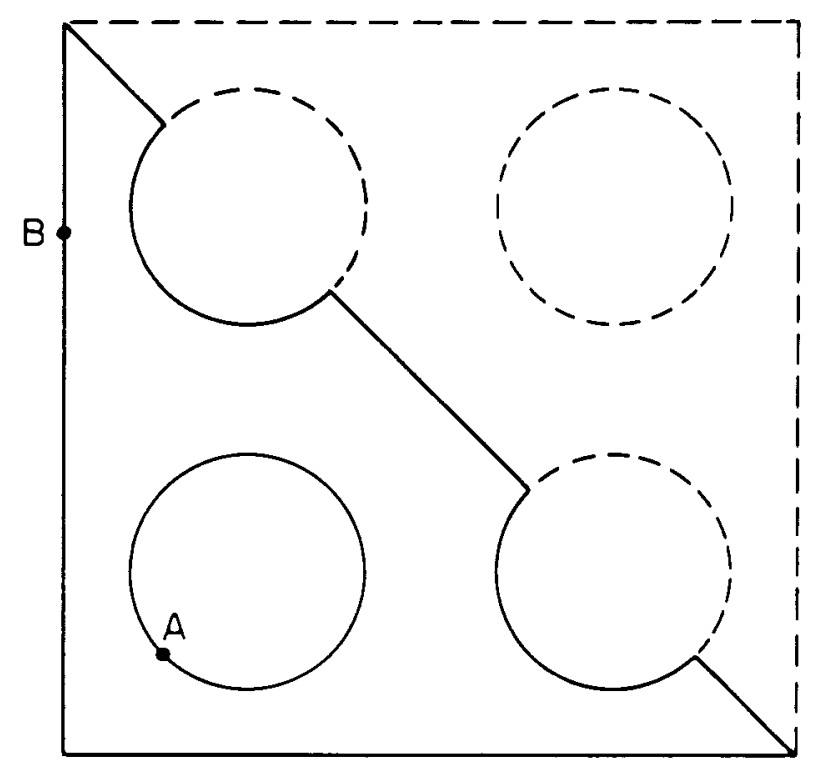

0
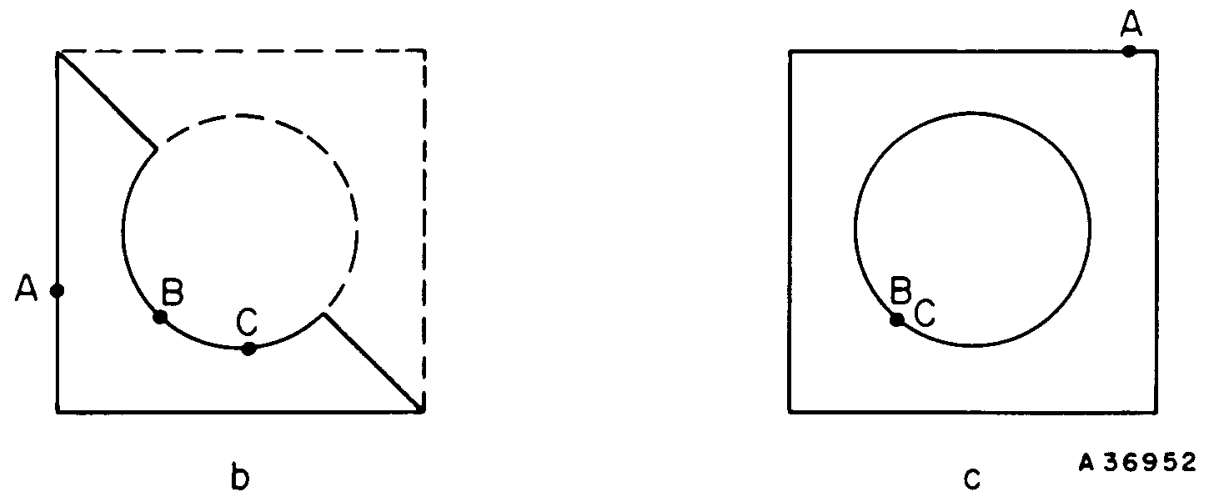

FIGURE 6. SHAPES CONSIDERED IN ANALYSIS 


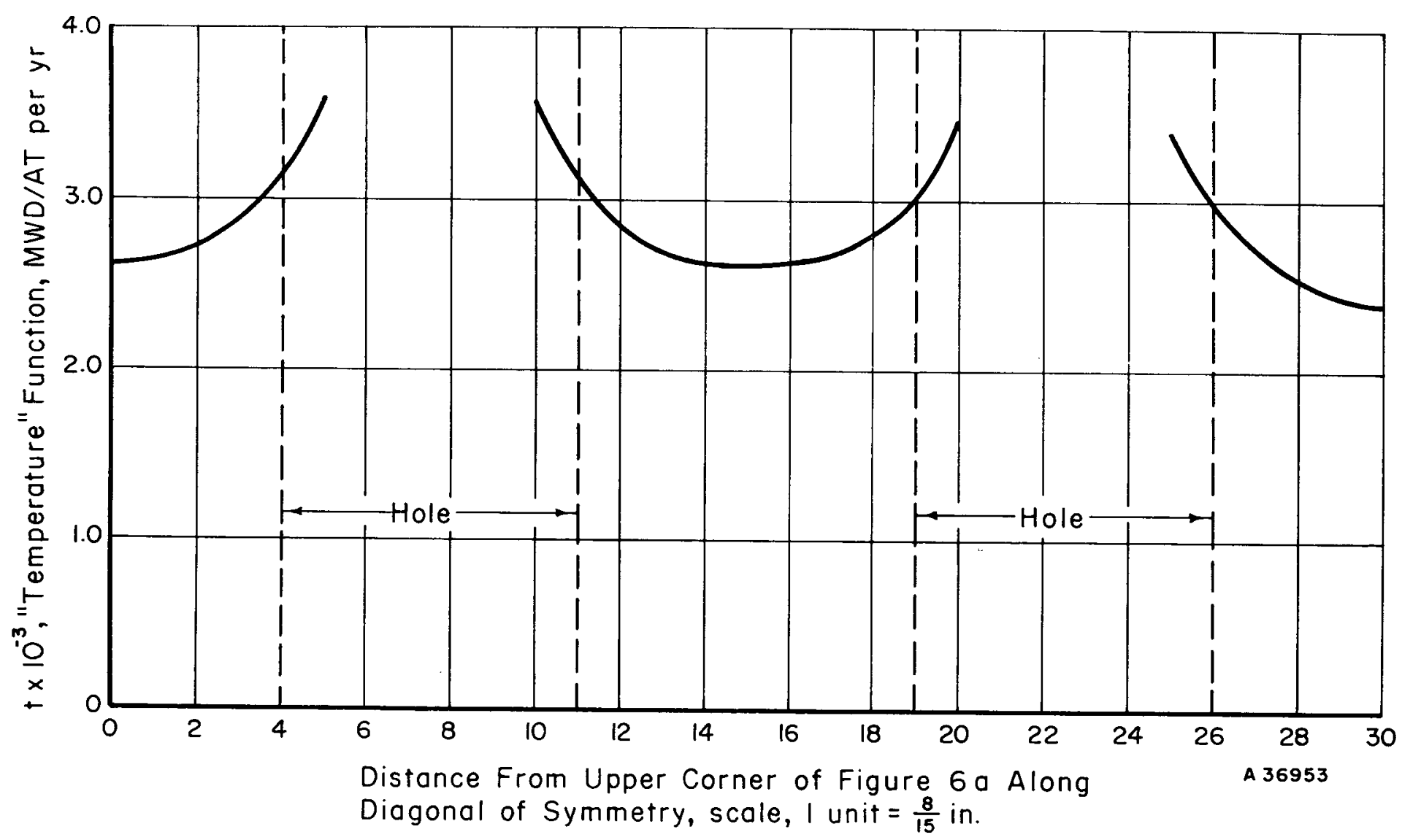

FIGURE 7. "TEMPERATURE" FUNC TION ALONG DIAGONAL 
TABLE 3. VALUE OF MAXIMUM TENSILE STRESS

\begin{tabular}{|c|c|c|}
\hline $\begin{array}{l}\text { Number of } \\
\text { Mesh Points }\end{array}$ & Point & $\begin{array}{c}\text { Maximum Tensile Stress } \\
\text { in xy-Plane, } \\
\text { psi }\end{array}$ \\
\hline 231 & A & 6800 \\
\hline 602 & B & 4300 \\
\hline
\end{tabular}

Thus, the maximum tensile stress and its location vary considerably as the mesh is refined. A further mesh refinement was not possible in this case as the number of equations would become prohibitively large. However, in the cases described later where a further mesh refinement was carried out, the change in the stresses was perhaps 10 per cent with the further mesh refinement. Thus, the results for the present problem would not be expected to change much with further mesh refinement.

Figures $6 \mathrm{~b}$ and $6 \mathrm{c}$ show the shapes considered when a subdivision of the body into quarters was made. The same "temperature" function used for Figure 6a was considered. Because the additional free surfaces afford some stress relief, it was expected that the maximum stresses would be less after the subdivision.

The shape of Figure 6b was considered with the "temperature" function corresponding to the lower right triangular portion of Figure 6a. The differences in the "temperature" function between this quadrant and the upper left quadrant were not considered to be significant enough to repeat the calculation with the different "temperature" function. Three mesh sizes were considered (87, 211 , and 563 mesh points, respectively). The locations of the maximum tensile stresses in the xy-plane are shown in Figure $6 \mathrm{~b}$ as points $A, B$, and $C$, respectively. Table 4 shows the magnitudes of the stress maxima.

\section{TABLE 4. VALUE OF MAXIMUM TENSILE STRESS}

\begin{tabular}{ccc}
\hline & & $\begin{array}{c}\text { Maximum Tensile Stress } \\
\text { in xy-Plane, } \\
\text { Number of } \\
\text { Mesh Points }\end{array}$ \\
\hline & Point & 2300 \\
21 & A & 1300 \\
563 & B & 1440 \\
\hline
\end{tabular}

Thus, the position of the maximum tensile stress migrates from the exterior boundary to the interior hole boundary. In addition, the value of the maximum tensile stress does not change much in the last mesh refinement as mentioned earlier. Again, the large change in the first mesh refinement is to be noted.

The shape of Figure 6c was then considered with the "temperature" function corresponding to the lower left quadrant of Figure 6a. Three mesh sizes identical to those considered previously were studied $(131,356$, and 1000 mesh points, respectively). The locations of the maximum tensile stresses in the xy-plane indicated in Figure $6 \mathrm{c}$ as Points $A, B$, and $C$, respectively. Table 5 shows the magnitudes of the stress maxima. 
TABLE 5. VALUE OF MAXIMUM TENSILE STRESS

\begin{tabular}{|c|c|c|}
\hline $\begin{array}{c}\text { Number of } \\
\text { Mesh Points }\end{array}$ & Point & $\begin{array}{c}\text { Maximum Tensile Stress } \\
\text { in xy-Plane, } \\
\text { psi }\end{array}$ \\
\hline 131 & A & 1480 \\
\hline 356 & B & 2340 \\
\hline 1000 & $\mathrm{C}$ & 2690 \\
\hline
\end{tabular}

Again, it is to be noted that the maximum stress migrates from the exterior boundary to the interior boundary. Also as before, the stress change in the last mesh refinement is small compared to that of the first mesh refinement.

Comparing the results from the three cases, it appears that by subdividing the graphite element the maximum tensile stress in the xy-plane can be reduced about 40 per cent. However, this is for a particular choice of constants and assumes the plane strain conditions are satisfied. The stresses reported here are for the parameter choices:

$$
\begin{aligned}
& \mathrm{B} 2=0.009273 \mathrm{~cm}^{-2} \\
& D=-6,086 \mathrm{psi} \text { per unit "temperature" } \\
& \lambda_{1}=0.20882 \\
& \lambda_{3}=0.47791 .
\end{aligned}
$$

The average "temperature" over the element considered is about 2.788 "temperature" units. A "temperature" unit corresponds to a graphite exposure of $1000 \mathrm{MWD} / \mathrm{AT}$, ton, a descriptive unit carried over from the experiments which measure the effects of the fast-neutron irradiation. It is sufficient to note that the "temperature" values used here relate to a possible exposure condition of the graphite elements in the center of the EGCR after 1 year of operation. The stress values, however, are based upon a 20-year exposure.

The plane-strain condition can only be met if bending moments are applied to the elements. The bending moments required after 20-year exposures were calculated and are given in Table 6.

TABLE 6. BENDING MOMENTS REQUIRED AFTER 20-YEAR EXPOSURES

\begin{tabular}{lll}
\hline & \multicolumn{2}{c}{ Bending Moments, lb-in. } \\
\cline { 2 - 3 } Element Shape & About x-Axis & About y-Axis \\
\hline Figure 6a & $-1.16 \times 10^{5}$ & $-9.22 \times 10^{4}$ \\
Figure 6b & $-1.60 \times 10^{4}$ & $-8.95 \times 10^{3}$ \\
Figure 6c & $-2.23 \times 10^{4}$ & $-7.46 \times 10^{3}$ \\
\hline
\end{tabular}

In addition to the bending moments, the displacements were calculated for each of the shapes considered. As an example, Figure 8 shows an exage ration of the distortion 
induced by the stresses for the shape of Figure 6c. It will be noted that the hole perimeter shortens as do the exterior boundaries. The latter also bulge in the middle.

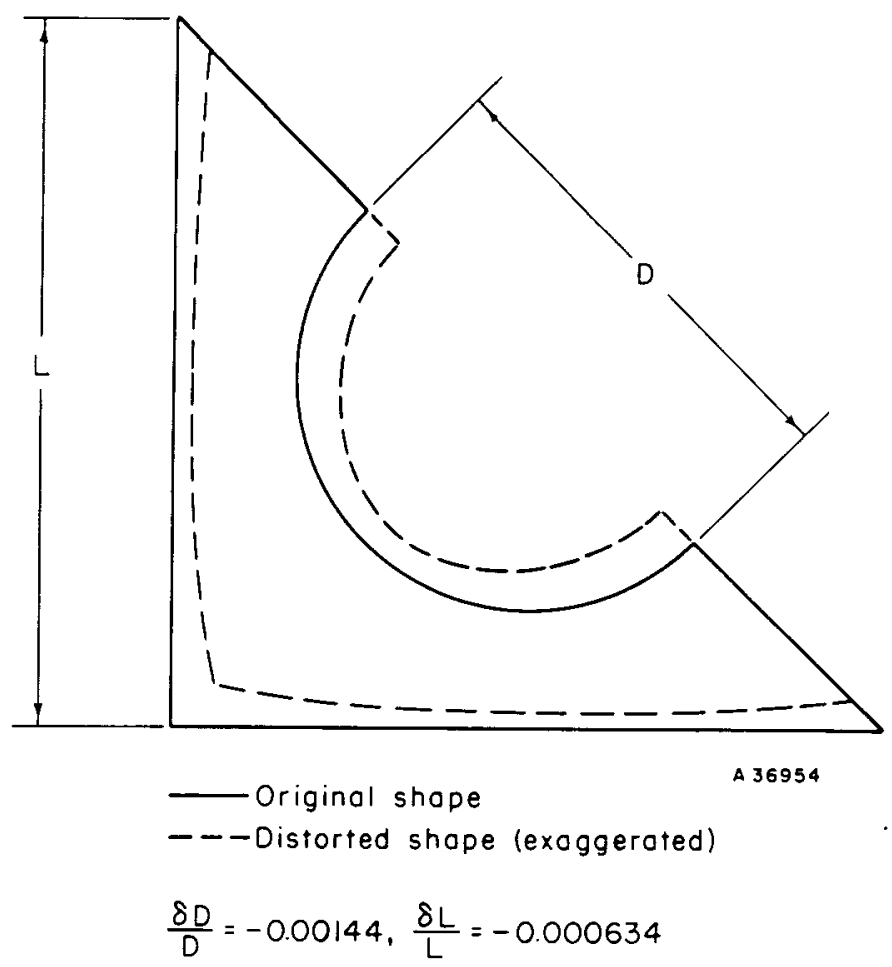

FIGURE 8. DISTORTION OF GRAPHITE ELEMENT

It is of interest to investigate how well the stress boundary conditions, Equation(7), are satisfied on the boundaries. It should be noted that the boundary conditions used, Equation (10), are mathematically equivalent but not numerically equivalent to Equation (7). It should further be noted that the boundary conditions are applied at boundary points which are not necessarily mesh points and that stresses are computed only at mesh points. Figures 9 and 10 show the stresses at the mesh points on the left edge of the shape in Figure 6c. From Figure 9, it will be noted that the fine mesh results show an oscillatory behavior of small amplitude. This behavior was not observed for the solutions for the other shapes and its explanation is not yet known. However, because of its small amplitude, it does not influence the stress values significantly. Both $\sigma_{\mathbf{x}}$ and $T_{\mathbf{x y}}$ should, of course be zero on the edge considered so Figures 9 and 10 indicate the deviations from the actual boundary conditions. As another illustration of the kind of convergence obtained, Table 7 gives the stresses at points in the shape of Figure 5 for the three different mesh spacings. The points are identified by the appropriate point numbers shown in Figure 5. It will be noted that the change in the stresses after the second mesh refinement is much smaller than that after the first mesh refinement. In addition, Point 7 shows a tendency to converge to the physical values. $\sigma_{x}=\sigma_{y}=\tau_{x y}=0$ at the corner. 


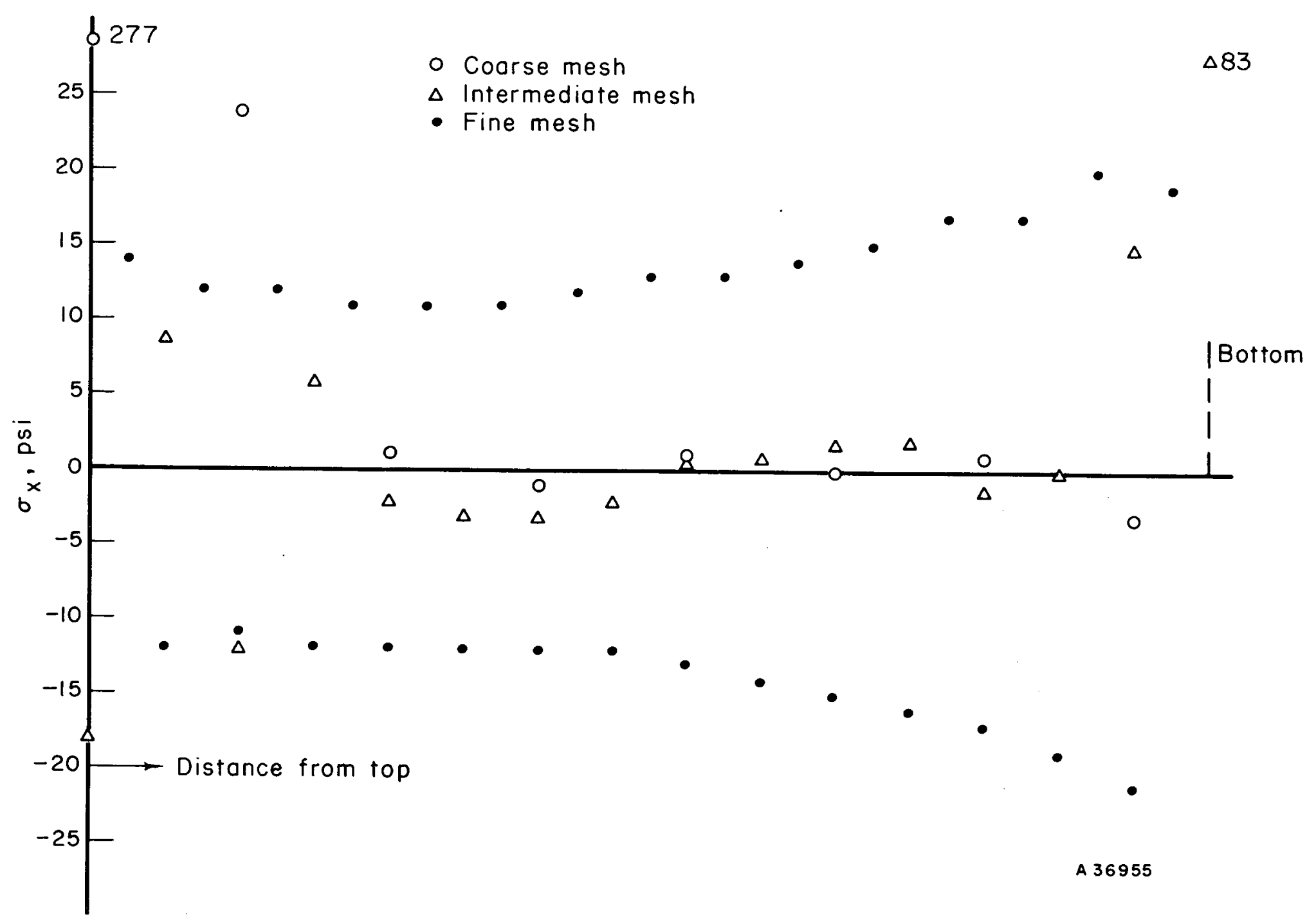

FIGURE 9. BOUNDARY STRESS ALONG LEFT EDGE OF SHAPE IN FIGURE 6c 


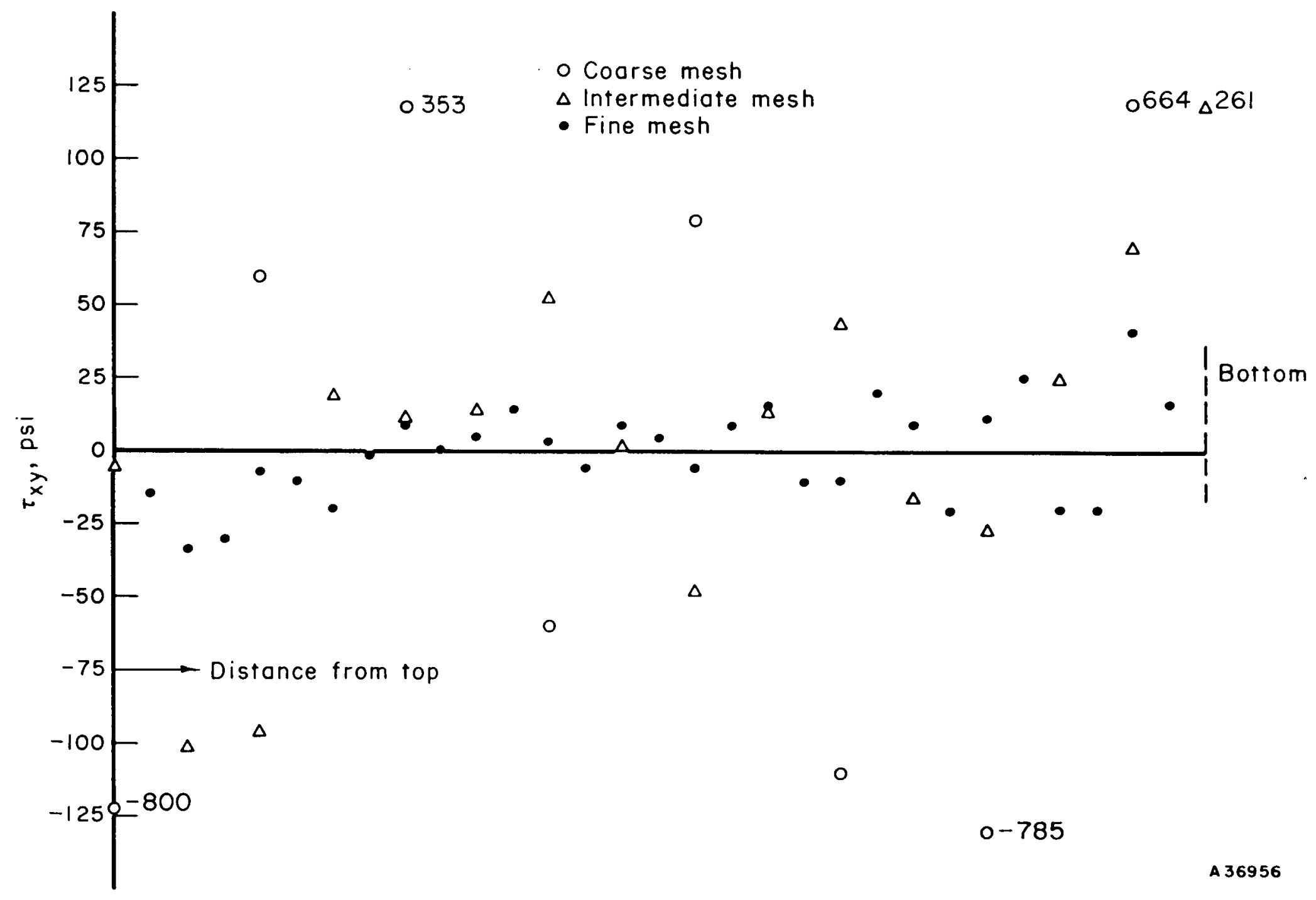

FIGURE 10. BOUNDARY STRESS ALONG LEFT EDGE OF SHAPE IN FIGURE 6c 
TABLE 7. STRESSES AT SELECTED POINTS FROM FIGURE 5 FOR THE THREE MESH SIZES

\begin{tabular}{|c|c|c|c|c|c|}
\hline $\begin{array}{l}\text { Point in } \\
\text { Figure } 5\end{array}$ & $\begin{array}{l}\text { Number of } \\
\text { Mesh Points } \\
\text { In Problem }\end{array}$ & $\begin{array}{l}\sigma_{x}, \\
\text { psi }\end{array}$ & $\begin{array}{l}\sigma_{y} \\
\text { psi }\end{array}$ & $\begin{array}{l}\tau_{x y}, \\
\text { psi }\end{array}$ & $\begin{array}{c}\sigma_{z}{ }^{(a)}, \\
\text { psi }\end{array}$ \\
\hline 7 & 87 & 278 & 278 & -610 & -749 \\
\hline 7 & 211 & 95 & 93 & -323 & -780 \\
\hline 7 & 563 & 33 & 33 & -9 & -896 \\
\hline 13 & 87 & -68 & -68 & -194 & -408 \\
\hline 13 & 211 & -166 & -163 & -46 & -402 \\
\hline 13 & 563 & -141 & -140 & -63 & -482 \\
\hline 20 & 87 & -87 & -87 & 164 & 1940 \\
\hline 20 & 211 & 443 & 438 & 534 & 2200 \\
\hline 20 & 563 & 542 & 541 & 568 & 2152 \\
\hline 50 & 87 & -390 & -16 & -106 & -427 \\
\hline 50 & 211 & -52 & -215 & 122 & -354 \\
\hline 50 & 563 & -44 & -147 & 130 & -427 \\
\hline
\end{tabular}

(a) Not true $\sigma_{z}$ but $\frac{\mathrm{D}}{\mathrm{E}_{\mathrm{z}} \alpha_{\mathrm{z}}} \sigma_{z}$.

These results indicate that further mesh refinement would not alter the stress values appreciably.

It can be concluded that the numerical procedures and computer codes described here offer a useful substitute to the tedious method of relaxation widely used in stress calculations. Further, the accuracy of these procedures should be as good or better than the method of relaxation for the same mesh-point array. In addition, the procedures and codes described here are quite general and can handle shapes and conditions which can be handled by relaxation only with great complication.

The results for the specific problems considered indicate that some advantage can be obtained by subdividing the graphite moderator elements in the manner being considered.

\section{REFERENCES}

(1) Redmond, R. F., Pollack, H., Klickman, A. E., Hogan, W. S., Epstein, H. M., and Chastain, J. W. , "Numerical Solution of Fuel-Element The rmal-Stress Problems", BMI-1422 (February 26, 1960).

(2) Kaiser Engineers and Allis Chalmers Mfg. Co., "Experimental Gas-Cooled Reactor, Preliminary Hazards Summary Report", ORO-196 with Supplement (May, 1959). 
(3) Stanek, F. J., "The Thermal Stress Equations in Plane Rectangular Coordinates for an Anisotropic Material", unpublished report, Oak Ridge National Laboratory, (April, 1960).

(4) Timoshenko, S., and Goodier, J. N., Theory of Elasticity, McGraw-Hill Book Company, Inc., 2nd edition, New York (1951).

(5) Southwell, R. V., Relaxation Methods in Theoretical Physics, Clarendon Press, Oxford (1956).

(6) Hildebrand, F. B., Introduction to Numerical Analysis, McGraw-Hill Book Company, Inc., New York (1956).

(7) F4-NY-GEL 1, "Solution of Simultaneous Linear Equations", SHARE Distribution Number 313, SHARE Reference Manual for the IBM-704.

RFR:LEH:RWC/nb 



\section{APPENDIX A}

MINIMIZATION OF RESIDUAL AS AN ITERATIVE METHOD 


\section{APPENDIX A}

\section{MINIMIZATION OF RESIDUAL AS AN ITERATIVE METHOD}

This method has been described previously(1) and hence only the essential features of the method are presented here.

Consider the matrix equation

$$
\mathrm{A} \phi=\mathrm{a},
$$

where

$$
\phi=\left(\begin{array}{c}
\phi_{1} \\
\phi_{2} \\
\cdot \\
\cdot \\
\phi_{\mathrm{R}}
\end{array}\right), \quad \mathrm{a}=\left(\begin{array}{c}
\mathrm{a}_{1} \\
\mathrm{a}_{2} \\
\cdot \\
\cdot \\
\mathrm{a}_{\mathrm{R}}
\end{array}\right),
$$

and $A$ is a nonsingular $R \times R$ matrix. The $\phi_{1}, \phi_{2}, \ldots, \phi_{R}$ are the unknowns to be determined for a given $A$ and $a$.

The residual vector is defined here by

$$
\mathrm{r}=\mathrm{A} \phi-\mathrm{a},
$$

and the length squared of $x$ is given by

$$
|r|^{2}=r \cdot r=|A \phi-a|^{2}=\sum_{i=1} r_{i}^{2} .
$$

Assume that an estimate for $\phi$ at some stage of the iteration is given by $\phi^{\mathrm{n}}(\mathrm{s})$, where

$$
\phi^{\mathrm{n}}(\mathrm{s})=\left(\begin{array}{c}
\phi_{1}^{\mathrm{n}+1} \\
\phi_{2}^{\mathrm{n}+1} \\
\cdot \\
\cdot \\
\phi_{\mathrm{s}-1}^{\mathrm{n}+1} \\
\phi_{\mathrm{s}}^{\mathrm{n}} \\
\cdot \\
\cdot \\
\phi_{\mathrm{R}}^{\mathrm{n}}
\end{array}\right)
$$

(1) References at end of Appendix. 
The nomenclature indicates that in the $\mathrm{n}^{\text {th }}$ iteration cycle the elements $\phi_{1}, \phi_{2}, \ldots$, $\phi_{S-1}$ have been iterated but the elements $\phi_{S}, \phi_{S+1}, \ldots, \phi_{R}$ have not been iterated as yet.

It is then desired to choose $\phi^{\mathrm{n}+\mathrm{l}}$ in such a way that $|\mathrm{r}|^{2}$ is minimized. To determine the way this choice must be made $\phi^{\mathbf{n}}(s+1)$ is put into Equation $(A-3)$ for $\phi$ and it is required that

$$
\frac{\partial|r|^{2}}{\partial \phi_{s}^{n+1}}=0
$$

which gives

$$
\frac{\partial|r|^{2}}{\partial \phi_{S}^{n+1}}=2 r \cdot \frac{\partial r}{\partial \phi_{S}^{n+1}}=2(A \phi-a) \cdot A \frac{\partial \phi}{\partial \phi_{s}^{n+1}}=0,
$$

where the right-side terms contain scalar products of $\mathrm{R}$-vectors. However, since only $\phi_{\mathrm{S}}^{\mathrm{n}+1}$ is being varied

$$
\begin{aligned}
\frac{\partial \phi_{r}^{\mathrm{n}}(\mathrm{s}+1)}{\partial \phi_{\mathrm{s}}^{\mathrm{n}+1}} & =0, \quad \mathrm{r} \neq \mathrm{s}, \\
& =1, \quad \mathrm{r}=\mathrm{s},
\end{aligned}
$$

and, therefore,

$$
\sum_{k}\left[A \phi^{n}(s+1)-a\right]_{k} A_{k s}=0
$$

or, in detail

$$
\sum_{\mathrm{k}}\left[\sum_{\ell} A_{k \ell} \phi_{\ell}^{\mathrm{n}}(\mathrm{s}+1)-\mathrm{a}_{\mathrm{k}}\right] \mathrm{A}_{\mathrm{ks}}=0 ;
$$

or, using the notation for the residual vector corresponding to $\phi_{\ell}^{\mathrm{n}}(s+1)$,

$$
\sum_{k} r_{k}^{n}(s+1) A_{k s}=0
$$

where

$$
r_{k}^{n}(s+1)=\sum A_{k} \phi_{\ell}^{n}(s+1)-a_{k}
$$

An expression can now be obtained from (A-6) by noting that

$$
r_{k}^{n}(s+1)=r_{k}^{n}(s)+A_{k s}\left[\phi_{s}^{n+1}-\phi_{s}^{n}\right]
$$


Hence,

$$
\phi_{\mathrm{s}}^{\mathrm{n}+1}=\phi_{\mathrm{s}}^{\mathrm{n}}-\frac{1}{\sum_{\mathrm{k}} \mathrm{A}_{\mathrm{ks}}^{2}} \sum_{\mathrm{k}} \mathrm{A}_{\mathrm{ks}} \mathrm{r}_{\mathrm{k}}^{\mathrm{n}}(\mathrm{s})
$$

This then is the new value of $\phi_{S}$ which minimizes the square of the residual vector. Since the vectors

$$
\left(\begin{array}{c}
A_{1 s} \\
A_{2 s} \\
\cdot \\
A_{R s}
\end{array}\right), s=1,2, \ldots, R
$$

are linearly independent ( $A$ is nonsingular) the minimization process will terminate only if Equation $(A-6)$ is satisfied for all $s$, it follows that there exists only the minimum given by the solution of $A \phi=a$. Hence, the iteration scheme will always converge to the true solution.

A method for improving the convergence has also been devised, called TerminalBlock Extrapolation. Let

$$
\delta \phi_{\mathrm{k}}^{\mathrm{n}}=\phi_{\mathrm{k}}^{\mathrm{n}+1}-\phi_{\mathrm{k}}^{\mathrm{n}}, \mathrm{k}=1,2, \ldots, \mathrm{R} .
$$

Define the new vector $\phi(K)$, where

$$
\phi_{k}(K)=\phi_{k}^{n+1}+K \delta \phi_{k}^{n}, k=1,2, \ldots, R \text {, }
$$

where $\mathrm{K}$ is to be determined so that the square of the residual vector $r(K)$ is minimized. Thus

$$
r(K)=A \phi(K)-a=r+K \delta r,
$$

where

$$
\begin{aligned}
r & =A \phi^{n+l}-a, \\
\delta r & =A \delta \phi^{n},
\end{aligned}
$$

and

$$
|r(K)|^{2}=r|2|+2 K r \cdot \delta r+K^{2}|\delta r|^{2}
$$


Clearly $r(K)^{2}$ is minimized if

$$
K=-\frac{r \cdot \delta r}{|\delta r|^{2}},
$$

and since $r \cdot \delta r<0, K>0$. Using this optimum choice of $K$ in the expression for $r^{2}(K)$ gives

$$
|r(K)|^{2}=|r|^{2} \sin ^{2} \theta
$$

where

$$
\cos \theta=\frac{r \cdot \delta r}{|r||\delta r|}
$$

Thus the Terminal-Block Extrapolation will reduce the residual vector significantly if the vectors $r$ and $\delta r$ are nearly parallel. Since $r, \delta r \phi$, and $\delta \phi$ are available from the normal iteration there is little additional arithmetic required to perform the extrapolation.

\section{$\underline{\text { Reference }}$}

(1) Redmond, R. F., Pollack, H., Klickman, A. E., Hogan, W. S., Epstein, H. M. , and Chastain, J. W. "Numerical Solution of Fuel-Element Thermal-Stress Problems", BMI-1422 (February 26, 1960). 
APPENDIX B

DETAILS OF COMPUTER CODES 


\section{APPENDIX B}

\section{DETAILS OF COMPUTER CODES}

\section{Code Description}

The codes described here have been developed for use on a $32 \mathrm{~K} \mathrm{IBM}-7090$ with a minimum of ten tape units. The codes described below can be used separately or serially with proper tape assignments.

\section{Coefficient-Matrix Routine (I)}

This program generates the coefficient matrix and the right-side vector which when solved will yield the stress-function solution. The matrix is generated from information concerning the geometry of the body and mesh setup. The program's input is:

A. Card Input (FORTRAN DIP)
(1) IE
(Fixed Point)
(2) IB
(Fixed Point)
(3) IC
(Fixed Point)
(4) ICUR
(Fixed Point)
(5) IQC
(Fixed Point)
(6) IBEG
(Fixed Point)
(7) IEND
(Fixed Point)
(8) MMAX
(Fixed Point)
(9) NMAX
(Fixed Point)
(10) IPMSN
(Fixed Point)
(11) ICN
(Fixed Point)
(12) IBDSN
(Fixed Point)
(13) IEXPNS
(Fixed Point)
(14) ITABC $\emptyset$
(Fixed Point)
(15) IBDSNA
(Fixed Point)
(16) MA
(Fixed Point)
(17) NA
(Fixed Point)
(18) $\mathrm{ZM}$
(Floating Point)
(19) ZN
(Floating Point)
(20) B2
(Floating Point)
(21) TEMP
(Floating Point)
(22) THET
(Floating Point)
(23) RA
(Floating Point)
(24) $\mathrm{SA}$
(Floating Point)
(25) XNA
(Floating Point)
(26) YNA
(Floating Point)

B. Binary Magnetic Tape

(1) None. 
The program's output is:

A. Output Tape

(1) Coefficient-Matrix Input

(2) Coefficient Matrix and its right-side vector

B. Binary Magnetic Tape

(1) Coefficient Matrix and its right-side vector on Logical 6

C. On-Line Printer gives error conditions if any arise.

Restrictions:

A. $\mathrm{IE} \leq 1000$.

Matrix-Transpose Routine (II)

This program transposes the coefficient matrix for use in the Iteration Routine. The program's input is:

A. Card Input (Normal FORTRAN Input)

(1) NR card columns $2-5 \quad \mathrm{xxxx}$

(2) $N \emptyset R \emptyset \quad " \quad " \quad 8-10 \quad \mathrm{xxx}$

(3) $\mathrm{N} \emptyset \mathrm{NZ} \quad$ " $113-15 \quad \mathrm{xxx}$

B. Binary Magnetic Tape

(1) Coefficient Matrix

(a) Obtained from Logical 6 of Coefficient-Matrix Routine

(b) Mounted as Logical 5 of Transpose Routine.

The program's output is:

A. Output Tape

(1) None

B. Binary Magnetic Tape

(1) Coefficient Matrix and its transpose on Logical 6

C. On-Line Printer gives error conditions if any arise. 
Restrictions:
A. $\quad \mathrm{NR} \leq 1000$
B. $N \emptyset R \emptyset(N \emptyset N Z) \leq 16500$
C. $N \emptyset \mathrm{R} \emptyset(\mathrm{N} \emptyset \mathrm{NZ}+3) \leq 17000$
D. $\mathrm{K}_{1} \leq 500$.

Iteration Routine (III)

This program iterates on an initial estimate of the stress-function vector to obtain a better approximation to the stress-function solution. The program's input is:

A. Card Input (FORTRAN DIP)
(1) IR
(Fixed Point)
(2) IP $\emptyset \mathrm{CT} \perp$ (Fixed Point)
(3) $\mathrm{N}_{l}$
(Fixed Point)
(4) $\mathrm{N}_{2}$
(Fixed Point)
(5) $\mathrm{N}_{3}$
(Fixed Point)
(6) $\mathrm{N}_{4}$ (Fixed Point)
(7) $\mathrm{N}_{5}$ (Fixed Point)
(8) ALPHA
(Floating Point)
(9) $X$ (Floating Point)
(10) PHI
(Floating Point, if initial solution is not computed from interpolation routine)

B. Binary Magnetic Tape

(1) Coefficient Matrix and its transpose

(a) Obtained from Logical 6 of Transpose Routine

(b) Mounted as Logical 6 of Iteration Routine

(2) PHI (if initial solution is computed from interpolation routine)

(a) Obtained from Logical 7 of Interpolation Routine

(b) Mounted as Logical 7 of Iteration Routine.

The program's output is:

A. Output Tape

(1) Iteration Input 
(2) Normal Printout when specified
(a) Solution vector
(b) Delta solution vector
(c) Residual vector
(d) Square of residual vector

B. Binary Magnetic Tape

(1) PHI, iterated solution vector on Logical 7

C. ON-Line Printer gives error conditions if any arise.

Restrictions:
A. $\quad \mathrm{IR} \leq 1000$
B. $\mathrm{K}_{1} \leq 500$
C. $\mathrm{K}_{2} \leq 13000$
D. $\mathrm{K}_{3} \leq 7000$

Stress-Calculation Routine (IV)

This program calculates the stress components at the interior mesh points from the stress-function vector. The program's input is:

A. Card Input (FORTRAN DIP)
(1) IE (Fixed Point)
(2) NMAX (Fixed Point)
(3) MMAX (Fixed Point)
(4) IPN (Fixed Point)
(5) IB (Fixed Point)
(6) IØGEL 1 (Fixed Point)
(7) IPMSN (Fixed Point)
(8) ITABC $\emptyset$ (Fixed Point)
(9) $\mathrm{V}$ (Floating Point)
(10) RO (Floating Point)
(11) TEMP (Floating Point)

B. Binary Magnetic Tape

(1) Stress-function vector

(a) Obtained from either Logical 7 of Iteration Routine or Tape Unit A5 of GEL 1 
(b) Mounted on Logical 7 of Stress-Calculation Routine if obtained from Iteration Routine, mounted as A6 if obtained from A5 of GEL 1.

The program's output is:

A. Output Tape

(1) Stress-Calculation Input

(2) Stress components at each interior mesh point

(3) Minimum and maximum stresses and their mesh coordinates

B. Binary Magnetic Tape

(1) None

C. On-Line Printer gives error conditions if any arise.

Restrictions:

A. $\quad E I \leq 1000$.

\section{Displacements Routine (V)}

The program's input is:

A. Card Input (Fortran DIP)
(1) IE
(Fixed Point)
(2) IC
(Fixed Point)
(3) ICUR
(Fixed Point)
(4) NMAX
(Fixed Point)
(5) MMAX (Fixed Point)
(6) ITABC $\emptyset$ (Fixed Point)
(7) TEMP (Fixed Point)
(8) NA (Fixed Point)
(9) MA
(Fixed Point)
(10) IBEG
(Fixed Point)
(11) IEND
(Fixed Point)
(12) RA
(Floating Point)
(13) SA
(Floating Point)
(14) XNA
(Floating Point)
(15) YNA
(Floating Point)
(16) $\mathrm{ZN}$
(Floating Point)
(17) $\mathrm{ZM}$
(Floating Point)
(18) EPS
(Floating Point)
(19) DELS
(Floating Point)
(20) AMDA
(Floating Point) 
B. Binary Magnetic Tape

(1) Stress function, PHI; TBAR

(a) Obtained from Logical 7 of Stress-Calculation Routine

(b) Mounted on Logical 7 of Displacements Routine.

The program's output is:

A. Output Tape

(1) Routine's Input

(2) Relative displacements

B. Binary Magnetic Tape

(1) None

C. On-Line Printer gives error conditions if any arise.

Interpolation Routine (VI)

This program uses a coarse-mesh stress-function solution to obtain an interpolated fine-mesh stress-function solution as an initial guess for the Iteration Routine. The interpolation formula used is obtained by making a double application of Stirling's interpolation formula and truncating the result. The program's input is:

A. Card Input (FORTRAN DIP)
(1) IE
(Fixed Point)
(2) NMAX
(Fixed Point)
(3) ITABC $\emptyset$
(Fixed Point)
(4) IFE
(Fixed Point)
(5) NFMAX
(Fixed Point)
(6) ITABFM
(Fixed Point)
(7) IEXPAN
(Fixed Point)
(8) ISM
(Fixed Point)
(9) MGøIPC
(Fixed Point)
(10) PHII
(Floating Point, if solution is not on tape)

B. Binary Tape Input

(1) Stress-function vector (PHI)

(a) Obtained either from Logical 7 of the Iteration Routine or Tape Unit A5 of GEL 1

(b) Mounted on Logical 6 of Interpolation Routine or obtained from Logical 7 of the Iteration Routine, mounted as A6 if obtained from A5 of GEL 1. 
The program's output is:

A. Output Tape

(1) Interpolation Input

(2) Interpolated stress function for fine mesh

B. Binary Magnetic Tape

(1) Interpolated stress function for fine mesh on Logical 7

C. On-Line Printer gives error conditions if any arise.

Restrictions:

IFE $\leq 1000$.

GEL 1 Routine (VII)

This program converts the coefficient matrix and its right side from a FORTRAN format to a format acceptable to NY GEL 1 , finds the direct solution to the coefficient matrix and its right side using the subroutine NY GEL 1, and outputs the solution on a decimal print tape. NY GEL 1 is a SHARE subroutine that finds the solution of simultaneous linear equations using Gaussian elimination with complete positioning for size. The computations are in double precision machine floating point. For details of subroutine see NY GEL 1 SHARE writeup, Distribution Number 313. The program's input is:

A. Card Input (Normal FORTRAN Input)

(1) IR Card columns $3-5 \mathrm{xxx}$

B. Binary Magnetic Tape

(1) Coefficient Matrix

(a) Obtained from Logical 6 of Coefficient-Matrix Routine

(b) Mounted on Tape Unit Bl

(2) Blank Tapes
(a) Mount on Al
(b) Mount on A2
(c) Mount on A3
(d) Mount on A4 


\section{(e) Mount on A5 \\ (f) Mount on B2.}

The program deck with IR data card at the end is read in on-line. The program is run under the 704-7090 compatibility package. The rightmost key on the console is placed down before the program is read in.

The program's output is:

A. Output Tape B2

(1) Solution vector PHI

(2) Residual vector

B. Binary Magnetic Tape

(1) A5 solution vector PHI.

\section{Restrictions:}

See SHARE write-up of NY GEL 1.

\section{Input Terminology}

\section{General Terms}

ALPHA - Residual minimizing factor $0<$ ALPHA $<2$

B2 - Constant in wave equation: $\nabla^{2} t-(B 2) t=0 \nabla^{2}=\frac{\partial^{2}}{\partial r^{2}}+\frac{\partial^{2}}{\partial s^{2}}$,

$$
\text { i. e., in mesh units) }
$$

IB - Number of exterior mesh points (see text of report)

IC - Number of boundary points (coincident end points counted twice)

ICUR - Total number of boundary curves on body

IE $\quad-$ Total number of mesh points

IFE - See IE (IFE is associated with the fine mesh for interpolation)

IØGEL 1 - Code--zero if stress-function input tape is from Iteration Routine; one if stress-function input tape is from GEL 1

IPN - Identification or problem number for Stress-Calculation Routine 
IPØCT 1 - Code--zero if initial solution vector is on cards; one if it is on magnetic tape

IQC - Code for selecting form of right side of biharmonic equation (see ICN in equation tables below)

IR - Total number of equations in the system $(=I E)$

Kl - Upper bound for number of nonzero coefficients in any equation

K2 - Total number of nonzero coefficients in all the equations not including the right-side vector

K3 - Total number of packed indices corresponding to the nonzero coefficients of all the equations including the right-side vector

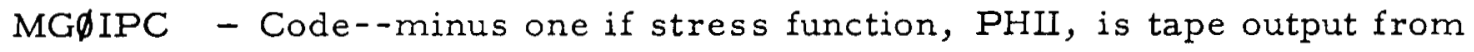
GEL 1; zero if stress function is tape output from Iteration Routine; plus one if stress function is on punched cards

MMAX - Number of rows in rectangle which covers mesh-point array

NFMAX - See NMAX (NFMAX is associated with the fine mesh)

NMAX - Number of columns in rectangle which covers mesh-point array

NøNZ - Maximum number of coefficients in any column of the coefficient matrix

$N \emptyset R \emptyset \quad-$ Number of rows of transpose to be found in one pass

NR - Order of the coefficient matrix to be transposed $=I E=I R$

$\mathrm{Nl} \quad-$ Specifies a normal printout every Nl iteration cycles

N2 - Specifies a normal printout every N2 extrapolation cycles

N3 - Specifies a stop in the program after N3 iteration cycles

N4 - Specifies an extrapolation after every N4 iteration cycles

N5 - Specifies a regeneration of residual vector every N5 extrapolation cycles

$\mathrm{v} \quad=\lambda_{1}=\beta_{3}\left(\alpha+\beta_{3}\right) /\left(\beta_{1}-\beta_{3}^{2}\right)$ 
$\mathrm{X}$

$=$ Program halts if square of residual vector is less than $\mathrm{X}$

$$
\begin{aligned}
& \text { IR } \\
& \sum_{i=1} r_{i}^{2}<x \\
& \mathrm{DDD}=\mathrm{D}=\mathrm{E}_{\mathrm{z}}\left(\alpha+\beta_{3}\right) \alpha_{\mathrm{z}} /\left(\beta_{1}-\beta_{3}^{2}\right) \\
& \text { EPS } \quad=\left(\alpha+\beta_{3}\right) \alpha_{z} \\
& A M D A=\lambda_{3} \\
& \mathrm{R} \phi=\text { Dimensionless parameter, output for } t \text { will be } t /(R \phi)^{2} \text {, } \\
& \mathrm{R} \phi=1.0 \text { if no scaling is desired. }
\end{aligned}
$$

\section{Boundary-Curves Tables}

IBEG - A table (ICUR entries) containing the beginning boundary-point number for each curve

IEND - A table (ICUR entries) containing the last boundary-point number for each curve

THET - A table (ICUR entries) containing the angle (radians) of rotation for each curve; the axis of symmetry is rotated to the y-axis, in a clockwise sense

$\mathrm{ZM} \quad-\mathrm{A}$ table (ICUR entries) containing the M-coordinate of the center for each hole in rectangular mesh units

ZN - A table (ICUR entries) containing the $\mathrm{N}$-coordinate of the center for each hole curve in rectangualr mesh units

DELS - A table (ICUR entries) containing the distance between boundary points in mesh units. 


\section{Correspondence Tables}

ITABC $\emptyset$ - Sequence of rectangular-array point numbers which coincide with the mesh points

ITABFM - See ITABC $\emptyset$ (this table is associated with the fine mesh).

\section{Miscellaneous Tables and Vectors}

IEXPAN (Interpolation Prog ram) $=0$ if fine mesh point coincides with coarse mesh point; if there is no coarse mesh point corresponding to the fine mesh point, IEXPAN gives the mesh-point number in fine mesh of the nearest interior point which does coincide with a coarse mesh point (IFE entries)

ISM (Interpolation Program) ISM $=0$ if fine mesh point does not coincide with a coarse mesh point; ISM = mesh point number in the coarse mesh if the point is common to both meshes (IFE entries)

PHI - Stress-function vector (IE values)

PHII - Stress-function vector associated with the coarse mesh (IE values)

TEMP - A table (IE entries) of temperature values associated with each mesh point; there exists a one-to-one correspondence between TEMP and ITABC $\emptyset$

\section{Geometry Tables}

IBDSNA - Boundary-point number

MA - M-value for interpolation center for boundary point

NA - N-value for interpolation center for boundary point

RA - R-value from interpolation center to boundary point

SA - S-value for interpolation center for boundary point

XNA $\quad-n_{x}$-value at boundary point

YNA $\quad-n_{y}$-value at boundary point.

\section{Equation Tables}

IPMSN - Mesh-point number of exterior point

IBDSN - Boundary point related to ICN number

IEXPNS - Interpolation point related to ICN number 
Type

1

2

3

4

5

6

7

8

9

10

11

12

Equation

$$
\nabla^{4} \phi=\left\{\begin{array}{r}
0, \text { IQC }=1 \\
1, \text { IQC }=2 \\
f(t), \text { IQC }=3 \\
(B 2) t, \text { IQC }=4
\end{array}\right.
$$

$$
\phi=0
$$

$$
\frac{\partial \phi}{\partial n}=0
$$

$$
\frac{\partial \nabla^{2} \phi}{\partial n}=0
$$

$$
\frac{\partial \phi}{\partial x}=0
$$

$$
\frac{\partial \phi}{\partial y}=0
$$

$\frac{\partial \phi}{\partial x}=\frac{\partial \phi}{\partial x}$ at first boundary point

$$
\frac{\partial \phi}{\partial y}=\frac{\partial \phi}{\partial y} \text { at first boundary point }
$$

$$
\int \frac{\partial}{\partial n} \nabla^{2} \phi d s=-\int \frac{\partial t}{\partial n} d s
$$

$\int\left[x \frac{\partial}{\partial n}-n_{x}\right] \nabla^{2} \phi d s=-\int\left[x \frac{\partial}{\partial n}-n_{x}\right]$ tds

$$
\int\left[y \frac{\partial}{\partial n}-n_{y}\right] \nabla^{2} \phi d s=-\int\left[y \frac{\partial}{\partial n}-n_{y}\right] t d s
$$

$\phi$ at mesh point $I=\phi$ at mesh point

$$
\text { IEXPNS (I) }
$$




\section{Execution Sequence of Routines to Solve Problem}

Each of the problems had a coarse mesh, intermediate mesh, and a fine mesh approximation. The routines were executed in the following sequence to solve the problems:

Routine

\begin{tabular}{ll}
\hline 1. Coefficient Matrix & $\begin{array}{l}\text { (coarse mesh) } \\
\text { (coarse mesh) }\end{array}$ \\
2. GEL 1 & (coarse mesh) \\
3. Stress Computations & (coarse to intermediate) \\
4. Interpolation & (intermediate mesh) \\
5. Coefficient Matrix & (intermediate mesh) \\
6. Matrix Transpose & (intermediate mesh) \\
7. Iteration & (intermediate mesh) \\
8. Stress Computation & (intermediate to fine) \\
9. Interpolation & (fine mesh) \\
10. Coefficient Matrix & (fine mesh) \\
11. Matrix Transpose & (fine mesh) \\
12. Iteration Routine & (fine mesh) \\
13. Stress Computation & (fine mesh) \\
14. Displacement Computation &
\end{tabular}

Computer System
G. E. FORTRAN
Compatibility
G. E. FORTRAN
G. E. FORTRAN
G. E. FORTRAN
G. E. FORTRAN
G. E. FORTRAN
G. E. FORTRAN
G. E. FORTRAN
G. E. FORTRAN
G. E. FORTRAN
G. E. FORTRAN
G. E. FORTRAN
G. E. FORTRAN

The MONITOR system and the FORTRAN input output subroutine IOU allow one to efficiently process the above sequence of routines.

With the MONITOR system one can stack G. E. FORTRAN routines and their card input one after another on an input tape. The only routine that cannot be controlled by MONITOR is GEL 1.

The IOU subroutine allows one to assign any FORTRAN logical tape number to any available tape drive (e.g., Logical 6 can be A5). Therefore, with the information given about which routines read and write on which FORTRAN logical tape units one can derive an efficient IOU subroutine for each routine so that intermediate tape handling will be a minimum. The above holds except for the following restrictions:

1. G. E. FORTRAN systems tape

2. Input tape

3. Output tape

4. Scratch tape

5. Scratch tape

6. Scratch tape

7. Scratch tape

8. Scratch tape

\section{Logical 1 Al}

Logical 2 A2

Logical 3 A3

A4

B 1

B2

B3

B4 


\section{G. E. FORTRAN System}

G. E. FORTRAN system is compatible with FORTRAN. However, G. E. FORTRAN has a flexible decimal input subroutine (DIP), MONITOR control, and a Format Generator. A detailed write-up of each is found in the G. E. FORTRAN manual.

DIP input contains a control punch in column one of the card. This control punch tells DIP how to convert the decimal input (i.e., floating point, fixed point, octal, etc.) and whether this card is the last card of this set of input. Control punch in Column 1:

$\begin{array}{ll}0 & \text { Octal instructions } \\ 2 & \text { Binary coded decimal stored in ascending order } \\ 3 & \text { Floating-point data } \\ 4 & \text { Fixed-point data } \\ 5 & \text { Binary coded decimal stored in decending order } \\ 3-8 & \text { Floating-point data - end of input } \\ 4-8 & \text { Fixed-point data - end of input }\end{array}$

Columns 2 to 72 contain the decimal data. Data words or fields may be of variable length. A field is terminated with either one comma or any number of blank spaces.

EXAMPLE (b represents a blank space)

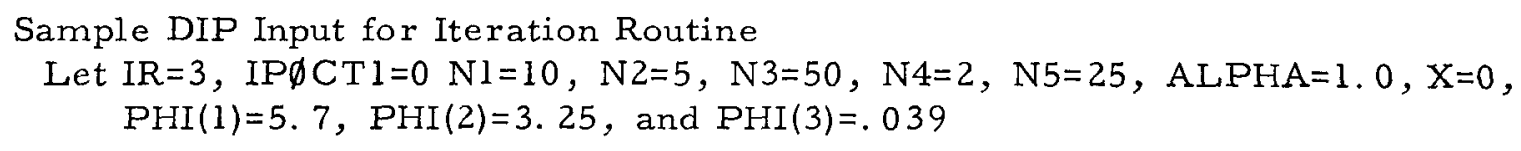

Card Column 12

4 IRb3bIP $\emptyset \mathrm{CT} 1 \mathrm{~b} 0 \mathrm{bN} 1 \mathrm{~b} 10 \mathrm{bN} 2 \mathrm{~b} 5 \mathrm{bN} 3 \mathrm{~b} 50 \mathrm{bN} 4 \mathrm{~b} 2 \mathrm{bN} 5 \mathrm{~b} 25$

$=$ ALPHAbl.0bXb0bPHIb5.7b-3.25b.039

The Format Generator provides a simplified way of writing a FøRMAT statement in the FORTRAN source language. One need not be concerned with it when processing production cases. Details are available in the G. E. FORTRAN manual.

The MONITOR control system will be discussed with respect to running a production problem. Other applications and details of MONITOR are available in the G. E. FORTRAN manual. In order to run a production problem one must process the set of routines in a specific sequence. Each routine with its MONITOR control cards (defined as a job) is written on the input tape in the particular sequence desired to process the problem.

The control cards associated with each routine are as follows:

(1) Identification Card

(a) Column 1 contains an

(b) Columns 7 to 72 contain descriptive information concerning the routine 


\section{B-15 and B-16}

(2) XEQ Card

(a) Column 1 contains a $\$$

(b) Columns 7 to 9 contain $X E Q$

(3) DATA Card

(a) Column I contains a $\$$

(b) Columns 7 to 10 contain DATA

The card sequence for a given job is then

(1) Identification Card

(2) XEQ Card

(3) FORTRAN Binary program and subroutines

(4) DATA Card

(5) DIP or FORTRAN data

The input tape would contain the following:
(1) First Job
(2) End of File
(3) Second Job
(4) End of File

..

$\cdot \cdot$

$N \quad$ Last Job

$\mathrm{N}+1$ End of File

$\mathrm{N}+2$ 1IDA Card Columns 1-4 IIDA

$\mathrm{N}+3$ End of File

Machine Setup

(1) Al G. E. FORTRAN systems tape

(2) A2 input tape

(3) A3 output tape

(4) A4 scratch tape

(5) BI scratch tape

(6) B2 scratch tape

(7) B3 scratch tape

(8) B4 scratch tape

(9) Any other blank tapes necessary in Routines (as controlled by the IOU

(10) Clear console keys, sense switches subroutine specification)

(11) Depress CLEAR, LOAD CARDS

(12) Read in on-line Binary START card. 
;

\{

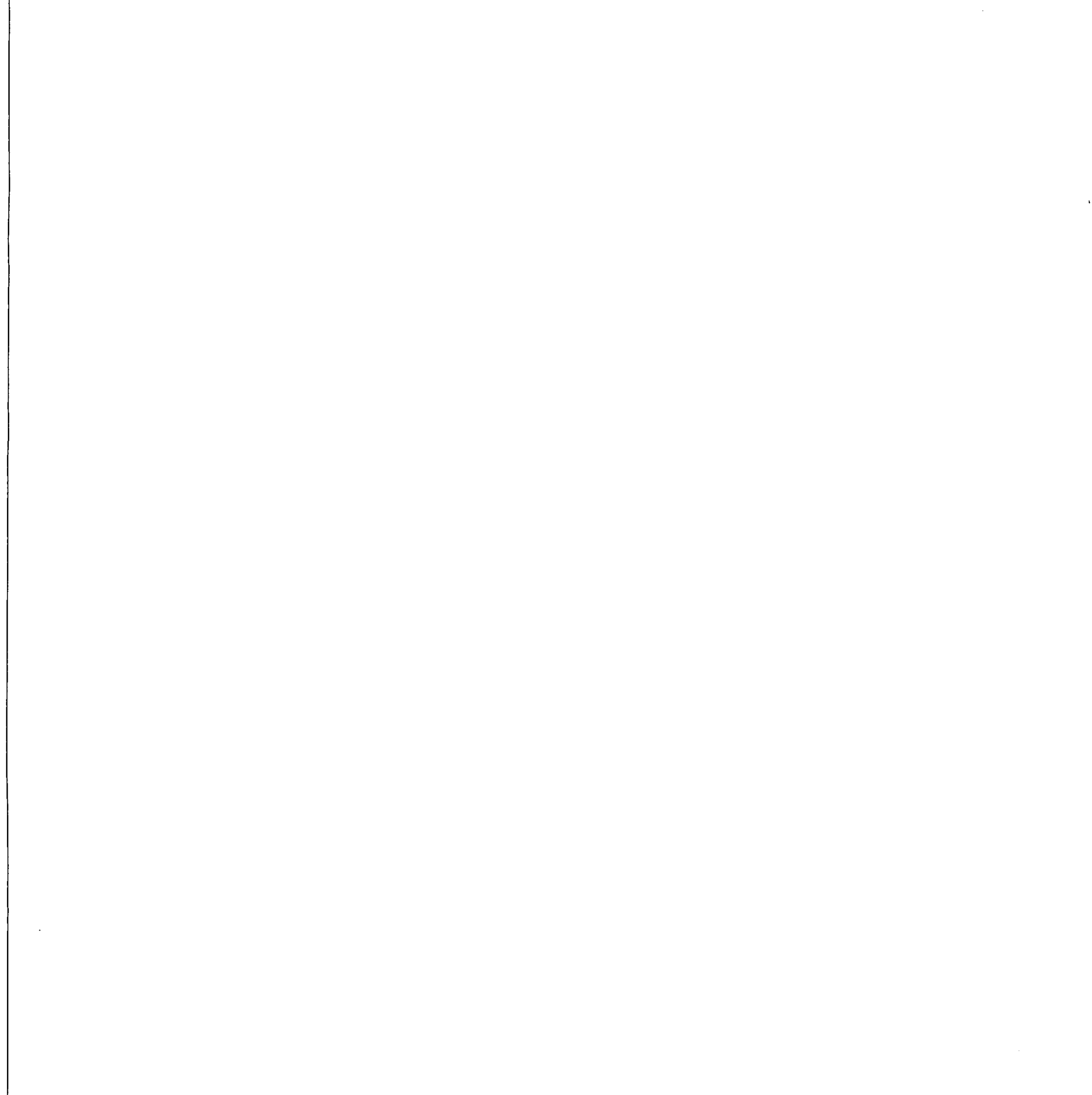


APPENDIX C

SAMPLE PROBLEM INPUT 


\section{APPENDIX C}

SAMPLE PROBLEM INPUT

The inputs pertinent to Figure 5 are presented here as an example.

IE $=87$
$I B=62$
$I C=26$
$I C U R=3$

$$
\begin{aligned}
& \text { MMAX }=12 \\
& \text { NMAX }=12 \\
& \text { IQC }=4
\end{aligned}
$$

Boundary-Curve Tables

$\begin{array}{cccccc}\text { I } & \text { IBEG } & \text { IEND } & \text { ZN } & \text { ZM } & \text { THET } \\ 1 & 1 & 10 & 6.75 & 6.75 & 0 \\ 2 & 11 & 20 & 6.75 & 6.75 & 0 \\ 3 & 21 & 26 & 6.75 & 6.75 & 0.785398\end{array}$

Boundary-Point Tables

Boundary

Point No.

RA

$\mathrm{SA}$

XNA

YNA

NA $\quad$ MA

1

2

3

4

5

6

7

8

9

10

11

12

13

14

15

16

17

18

19

20

21

22

23

24

25

26
0.500000

$-0.333330$

$-0.166667$

0 .

0.166667

$-0.6666667$

$-0.500000$

$-0.333333$

$-0.166667$

0 .

0 .

0 .

0 .

0 .

0 .

0 .

0 .

0 .

0 .

0 .

0.009846

0.319366

0.557280

0.632759

0.134964

$-0.509846$
$-0.500000$

$-0.500000$

$-0.500000$

$-0.500000$

$-0.500000$

$-0.500000$

$-0.500000$

$-0.500000$

$-0.500000$

$-0.500000$

$-0.500000$

0.333333

0.166667

0 .

$-0.166667$

0.666667

0.500000

0.333333

0.166667

0 .

$-0.009846$

0.634964

0.132759

0.057280

0.819366

0.509846
0.

0 .

0 .

0 .

0 .

0 .

0 .

0 .

0 .

0 .

$-1.000000$

$-1.000000$

$-1.000000$

$-1.000000$

$-1.000000$

$-1.000000$

$-1.000000$

$-1.000000$

$-1.000000$

$-1.000000$

0. 707110

0.987690

0.891010

0.453990

$-0.156430$

$-0.707110$

$-1.000000$

$10 \quad 10$

$-1.000000$

10

$-1.000000$

10

$-1.000000$

10

$-1.000000$

$-1.000000$

10

$-1.000000$

$-1.000000$

$-1.000000$

$-1.000000$

0 .

0 .

0 .

0 .

0 .

0 .

0 .

0 .

0 .

0 .

$-0.707110$

$-0.156430$

0.453990

0.891010

0.987690

0. 707110

10

$$
8
$$$$
7
$$

$$
\begin{aligned}
& 7 \\
& 6
\end{aligned}
$$$$
10
$$$$
\begin{array}{ll}
3 & 10 \\
3 & 10
\end{array}
$$$$
39
$$$$
38
$$$$
3 \quad 7
$$$$
\begin{array}{ll}
3 & 7
\end{array}
$$$$
36
$$$$
35
$$$$
\begin{array}{ll}
3 & 4 \\
3 & 3
\end{array}
$$$$
\begin{array}{ll}
3 & 3 \\
5 & 5
\end{array}
$$$$
\begin{array}{ll}
5 & 5 \\
4 & 7
\end{array}
$$$$
\begin{array}{ll}
4 & 7
\end{array}
$$$$
\begin{array}{ll}
4 & 8 \\
5 & 9
\end{array}
$$$$
7 \quad 10
$$$$
9
$$$$
9
$$ 
Exterior-Point Tables

\begin{tabular}{|c|c|c|c|c|c|c|c|}
\hline IPMSN & $\mathrm{ICN}$ & IBDSN & IEXPNS & IPMSN & ICN & IBDSN & IEXPNS \\
\hline 1 & 12 & 1 & 5 & 44 & 11 & 21 & 55 \\
\hline 2 & 6 & 20 & 7 & 45 & 12 & 33 & 54 \\
\hline 3 & 12 & 3 & 6 & 46 & 12 & 34 & 65 \\
\hline 4 & 12 & 4 & 11 & 47 & 5 & 13 & 49 \\
\hline 5 & 5 & 19 & 12 & 48 & 6 & 13 & 49 \\
\hline 6 & 6 & 19 & 12 & 52 & 7 & 25 & 64 \\
\hline 8 & 12 & 7 & 12 & 53 & 7 & 26 & 55 \\
\hline 9 & 12 & 8 & 18 & 56 & 12 & 39 & 66 \\
\hline 10 & 5 & 18 & 18 & 57 & 12 & 40 & 77 \\
\hline 11 & 6 & 18 & 18 & 58 & 5 & 12 & 60 \\
\hline 14 & 12 & 11 & 19 & 59 & 6 & 12 & 60 \\
\hline 15 & 12 & 12 & 26 & 68 & 12 & 43 & 78 \\
\hline 16 & 5 & 17 & 25 & 69 & 12 & 44 & 87 \\
\hline 17 & 6 & 17 & 25 & 70 & 5 & 11 & 61 \\
\hline 21 & 12 & 15 & 27 & 71 & 2 & 46 & 62 \\
\hline 22 & 12 & 16 & 33 & 72 & 5 & 9 & 61 \\
\hline 23 & 5 & 16 & 31 & 73 & 5 & 8 & 62 \\
\hline 24 & 6 & 16 & 31 & 74 & 5 & 7 & 63 \\
\hline 27 & 8 & 22 & 32 & 75 & 5 & 5 & 64 \\
\hline 28 & 9 & 21 & 20 & 76 & 5 & 4 & 65 \\
\hline 29 & 5 & 15 & 31 & 77 & 5 & 3 & 66 \\
\hline 30 & 6 & 15 & 31 & 78 & 5 & 2 & 67 \\
\hline 33 & 8 & 23 & 40 & 79 & 5 & 1 & 67 \\
\hline 34 & 7 & 22 & 32 & 80 & 6 & 9 & 61 \\
\hline 35 & 12 & 25 & 43 & 81 & 6 & 8 & 62 \\
\hline 36 & 12 & 26 & 53 & 82 & 6 & 7 & 63 \\
\hline 37 & 5 & 14 & 39 & 83 & 5 & 6 & 64 \\
\hline 38 & 6 & 14 & 39 & 84 & 6 & 5 & 64 \\
\hline 41 & 8 & 24 & 51 & 85 & 6 & 4 & 65 \\
\hline 42 & 7 & 24 & 51 & 86 & 6 & 3 & 66 \\
\hline 43 & 8 & 25 & 64 & 87 & 6 & 2 & 67 \\
\hline
\end{tabular}

$\underline{\text { ITABC } \emptyset \text { Table }}$

Physical

Mesh No.
$\operatorname{ITABC} \emptyset$

3

14

15

16

25

26
Physical

Mesh No. ITABC $\emptyset$

$\begin{array}{rr}7 & 27 \\ 8 & 28 \\ 9 & 29 \\ 10 & 37 \\ 11 & 38 \\ 12 & 39\end{array}$

Physical

MeshNo. ITABC $\emptyset$

13
14
15
16
17
18

13

40

41

42

49

50

51 
$C-3$ and C- 4

$\begin{array}{cc}\text { Physical } & \\ \text { Mesh No. } & \text { ITABC } \emptyset \\ 19 & 52 \\ 20 & 53 \\ 21 & 54 \\ 22 & 55 \\ 23 & 61 \\ 24 & 62 \\ 25 & 63 \\ 26 & 64 \\ 27 & 65 \\ 28 & 66 \\ 29 & 73 \\ 30 & 74 \\ 31 & 75 \\ 32 & 76 \\ 33 & 77 \\ 34 & 78 \\ 35 & 80 \\ 36 & 81 \\ 37 & 85 \\ 38 & 86 \\ 39 & 87 \\ 40 & 88 \\ 41 & 89\end{array}$

Physical

MeshNo. ITABC $\emptyset$

42

43

44

45

46

47

48

49

50

51

52

53

54

55

56

57

58

59

60

61

62

63

64
90

91

92

93

94

97

98

99

100

101

102

103

104

105

106

107

109

110

111

112

113

114

115
Physical

MeshNo. ITABC $\emptyset$

65

66

67

68

69

70

71

72

73

74

75

76

77

78

79

80

81

82

83

84

85

86

87
116

117

118

119

120

122

123

124

125

126

127

128

129

130

131

135

136

137

138

139

140

141

142 